CERN-TH/96-361

IASSNS-96/127

EHU-FT/9701

hep-th/9701142

\title{
Dilute D-Instantons at Finite Temperature
}

\author{
J. L. F. BARBÓN \\ Theory Division CERN \\ CH-1211 Geneva 23, Switzerland \\ barbon@mail.cern.ch \\ M. A. VÁzquez-Mozo \\ Institute for Advanced Study \\ Princeton, NJ 08540, U.S.A. \\ and \\ Dept. de Física Teórical \\ Universidad del País Vasco \\ Apdo. 644, E-48080 Bilbao, Spain \\ wtbvamom@lg. ehu.es
}

\begin{abstract}
We discuss Dirichlet instanton effects on type-IIB string thermodynamics. We review some general properties of dilute D-instanton gases and use the low-energy supergravity solutions to define the normalization of the instanton measure, as well as the effects of longrange interactions. Thermal singularities in the single-instanton sector are due to tachyonic winding modes of Dirichlet open strings. Purely bosonic D-instantons induce in this way hard infrared singularities that ruin the weak-coupling expansion in the microcanonical ensemble. However, type-IIB D-instantons, give smooth contributions at the Hagedorn temperature, and the induced mass and coupling of the axion field are insufficient to change the first-order character of the phase transition in the mean field approximation. $01 / 97$
\end{abstract}

1 Address after January 1st, 1997. 


\section{Introduction}

Since its prehistoric days, string theory has been formulated in a perturbative version in which the string coupling constant is assumed to be small. However, the shortcomings of this approach were early realized, since a great deal of physically interesting situations would involve some kind of non-perturbative phenomena. In fact, one could say that a weakly coupled description of the physics at the string scale needs non-perturbative input to be predictive at all, because of the non-renormalization theorems implied by supersymmetry, which is itself a necessary ingredient for consistency of the weakly coupled description. In spite of this, non-perturbative string physics has remained an elusive realm where no significative progress was made at a quantitative level, until the recent advent of string dualities (for a review see [1]). One of the lessons to be learned from this second string revolution is the dynamical relevance of generalized soliton states in string theory. Since these states have to decouple in the weak-coupling limit, their masses scale with inverse powers of the string coupling constant. In the case of type-II strings, string duality implies the existence of non-perturbative states carrying $\mathrm{R}-\mathrm{R}$ charge and having masses of the order of $\lambda^{-1}$. It was realized by Polchinski [2] that those states are provided by D-branes [3].

On general grounds, a $p$-brane with a tension of order $1 / \lambda$ produces non-perturbative effects of order $e^{-V_{p+1} / \lambda}$, where $V_{p+1}$ is a characteristic Euclidean world-volume associated semiclassically to a $(p+1)$-dimensional non-contractible cycle in target space. This means that, for the first time 四, we have an explicit semiclassical construction of the "stringy" non-perturbative effects already expected on the basis of perturbation theory estimates [5]. The D-brane description provides a perturbative treatment of the collective coordinate dynamics, something very problematic in other approaches to soliton quantization in string theory. It is then very interesting to understand the weak-coupling quantization of such objects, non only for the applications to duality, but more generally because they apply to more realistic situations without extended supersymmetry.

Among different $p$-dimensional D-branes, D-instantons $(p=-1)$ are special for several reasons. In spite of their stringy origin, they appear to be point-like objects when probed with closed strings at high energies, contrary to higher-dimensional D-branes, which have an effective size of order $\sqrt{\alpha^{\prime}}$ [6]. Another important point is the fact that the corresponding $e^{-1 / \lambda}$ effects are present in the ten-dimensional type-IIB theory while all the rest of Euclidean D-brane instantons are suppressed in the decompactification limit. As 
a result, they represent the technically simplest case. At a dynamical level, D-instantons differ from more conventional Yang-Mills instantons in that they do not have size moduli and, more importantly, there are long-range Coulomb interactions between instantons and anti-instantons, in complete analogy with the well-known monopole instantons producing a mass gap in $2+1$ gauge theories [7].

In the present paper we will be concerned with the physical consequences of Dinstanton effects in the thermodynamics of ten-dimensional type-IIB strings2. String theory at finite temperature [9] is one scenario in which non-perturbative effects have frequently been invoked to account for a number of perturbative riddles. Probably the most interesting of these is the existence of a critical temperature (the so-called Hagedorn temperature) at which the canonical description of the string collective breaks down. In switching from the canonical to the microcanonical ensemble a phase of negative specific heat appears above the critical temperature, indicating an instability of the string gas 3 . This Hagedorn "crisis" has been the subject, over the years, of much controversy as to whether it really signals a phase transition into a, yet mysterious, high-temperature phase of string theory or it just marks a maximum temperature of the string gas (for a recent entry, see [10]).

Generally speaking, instanton effects can dominate at weak coupling only when considering quantities without any perturbative contributions. Since this is not the case for the usual thermodynamical functions, such as the canonical free energy or the microcanonical entropy function, we do not expect important modifications of the perturbative lore at a qualitative level. In this sense, we discuss instanton-driven thermal singularities in the corresponding Dirichlet string diagrams as a check of the consistency of the D-instanton weak-coupling expansion.

Within the canonical approach, we find that the possible instanton singularities are hard but located well inside the Hagedorn domain, in a region of temperatures where the string diagram expansion does not make much sense. We have also studied the microcanonical description of the string gas near the Hagedorn transition, including D-instanton

2 Effects of Dirichlet boundaries on thermal strings were considered in the past by M. Green with a different motivation [8].

3 We are describing here the generic situation. If all spatial dimensions are compactified the negative specific heat phase disappears and the Hagedorn temperature becomes a maximum temperature both in the canonical and microcanonical ensembles. 
contributions to the density of states, computed as an inverse Laplace transform of the analytically continued canonical partition function. In this case the temperature singularities beyond the Hagedorn domain do have a concrete physical effect, as subleading terms in a high-energy expansion. Our results show that whenever new instanton-driven singularities appear, as in the case of purely bosonic strings, the whole instanton expansion makes no sense at all, being even more singular than could have been expected on the basis of the zero-temperature instabilities of bosonic strings. This instability is characterized by uncontrollable power corrections in the high-energy asymptotics of the microcanonical density of states. On the other hand, for type-IIB strings in ten dimensions, with a well-behaved perturbation theory, we find that D-instantons do not induce new singularities, and the Hagedorn phase transition seems to be triggered by the same tachyonic winding mode as in standard perturbation theory.

A genuine instantonic effect in the type-IIB theory is the generation of a nonperturbative mass and non-derivative couplings for the axion scalar field, protected in perturbation theory by a Peccei-Quinn symmetry. This is a situation where instantons can in principle produce qualitatively important effects through the axion dynamics. We have re-examined the role of the axion in the Atick-Witten mean field theory analysis of the Hagedorn phase transition and found that, while D-instantons tend to induce a second-order transition, the dynamics is still dominated by the dilaton, which produces a first-order phase transition with a critical temperature smaller than the Hagedorn temperature.

We will not consider in this paper the case of type-IIA strings. Due to the presence of the thermal non-contractible circle, Euclidean world-lines of Dirichlet 0-branes produce Wilson-Polyakov loop contributions of order $e^{-C \beta / \sqrt{\alpha^{\prime}} \lambda}$, which are interesting to study (see [11] for work in this direction). The T-duality that should relate the IIA and IIB cases works differently with thermal boundary conditions [12]. In any case, temperature T-duality (also called $\beta$-duality) is probably unphysical and should be broken.

The plan of the paper is as follows: we begin Section 2 by reviewing some general facts about D-instantons and the construction of ref. [13] of D-instanton solutions in typeIIB supergravity. After this we apply the collective coordinates method [14 to obtain the instanton measure and compute the effective action for the massless scalars (axion and dilaton) induced by the long-range Coulomb interactions. In Section 3 we start our 
discussion of D-instanton effects in string thermodynamics by studying the D-instantoninduced singularities in the canonical free energy and its relevance in the microcanical description of strings near the Hagedorn temperature. Section 4 will be devoted to the study of the relevance of D-instantons in the low-energy effective theory description of the Hagedorn transition. Finally, in Section 5 we will summarize our conclusions. A discussion of fermionic collective coordinates is included in Appendix A. For the sake of completeness, we will discuss the computation of the microcanonical density of states in perturbation theory in Appendix B, while in Appendix C we will review the construction of higher-order amplitudes in open bosonic string theory.

\section{Dirichlet Instanton generalities}

Formally speaking, D-instantons correspond to the "degenerate" case of $p=-1$ Dbranes, i.e. the world-volume is a point in space-time and the collective dynamics reduces to a finite-dimensional integral over moduli space. The action and interactions are determined by a theory of open strings without translational zero modes, and it was originally obtained as a T-dual of standard open strings with respect to all translational isometries.

A complete perturbative expansion for a dilute D-instanton gas was obtained in [15], [4] on the basis of T-duality and cluster decomposition. The complete partition function takes the form

$$
\mathcal{Z}=\sum_{n_{+}, n_{-}=0}^{\infty} \frac{1}{n_{+} ! n_{-} !} \prod_{j=1}^{n_{+}} \int d \mu_{j}^{+} \prod_{k=1}^{n_{-}} \int d \mu_{k}^{-} e^{-S_{\left(n_{+}, n_{-}\right)}}
$$

where $d \mu^{ \pm}$denotes the collective coordinates measure for a single D-instanton and the action in the $\left(n_{+}, n_{-}\right)$instanton sector is

$$
S_{\left(n_{+}, n_{-}\right)}=\Gamma_{0}+\sum_{j} \Gamma_{j}+\sum_{k} \Gamma_{k}+\sum_{\left(j_{1}, j_{2}\right)} \Gamma_{\left(j_{1}, j_{2}\right)}+\sum_{\left(k_{1}, k_{2}\right)} \Gamma_{\left(k_{1}, k_{2}\right)}+\sum_{(j, k)} \Gamma_{(j, k)}+3 \text { body; }
$$

here the index $j$ refers to instantons and $k$ to anti-instantons. We have an expansion in irreducible many-body interaction terms, each of them given by the sum of connected string diagrams with a number of boundaries attached to instantons, anti-instantons, or both. Specifically,

$$
\Gamma_{\left(j_{1}, \cdots ; k_{1}, \cdots\right)}=\sum_{g=0}^{\infty} \sum_{N_{1}^{+}, \ldots=0}^{\infty} \sum_{N_{1}^{-}, \ldots=0}^{\infty} \frac{\lambda^{2 g+\sum N^{+}+\sum N^{-}-2}}{N_{1}^{+} ! \cdots N_{1}^{-} ! \cdots} W\left(g, N_{j}^{+}, N_{k}^{-}\right) .
$$


Here $N^{ \pm}$denote the numbers of boundaries attached to the same instanton or antiinstanton, and $\lambda$ stands for the string coupling constant. The first term $\Gamma_{0}$ is the standard perturbative sum of string diagrams in vacuo, and the bare instanton action is given by $\Gamma_{ \pm}=\frac{|Q|}{\lambda}$, where $|Q|=W_{0,1}$ is numerically equal to the disk amplitude with Dirichlet boundary conditions. Diagrams without handles correspond to classical interactions between the instantons, the leading one coming from the cylinder diagram, which corresponds at low energies to the interactions induced by the overlap of the instanton tails at long distances (see Appendix A for an explicit expression). The expansion (2.3) and (2.1) provides a complete perturbative treatment of the instanton interactions, including the purely classical ones, giving a stringy version of a perturbative constrained instanton expansion [16], [17.

In an operator formalism we can translate the Dirichlet boundary conditions on the world-sheet fields into boundary states constructed as convenient coherent states in the single-string Hilbert space. The simplest description of such states in the supersymmetric case is achieved in the light-cone frame, using the Green-Schwarz formalism. In the notation and conventions of [18], the bosonic (anti-) D-instanton boundary state is a solution of the constraints $\left(\alpha_{n}^{i}-\bar{\alpha}_{-n}^{i}\right)\left|I_{ \pm}, p\right\rangle=0,\left(S_{n}^{a} \pm i \bar{S}_{-n}^{a}\right)\left|I_{ \pm}, p\right\rangle=0$ and may be written as the coherent state

$$
\left|I_{ \pm}, p\right\rangle=\exp \sum_{n=1}^{\infty}\left(\frac{\alpha_{-n}^{i} \bar{\alpha}_{-n}^{i}}{n} \mp i S_{-n}^{a} \bar{S}_{-n}^{a}\right)\left|0_{ \pm}, p\right\rangle
$$

where $S^{a}$ are Green-Schwarz fermions, transforming in the $\mathbf{8}_{\mathbf{s}}$ of $S O(8)$, the transverse rotation group. The ground states in (2.4) are the standard massless scalars of the type-IIB string $\left|0_{ \pm}, p\right\rangle=\frac{1}{4}(|p\rangle|i\rangle|\bar{i}\rangle \mp i|p\rangle|\dot{a}\rangle|\overline{\dot{a}}\rangle)$ satisfying $\left\langle 0_{ \pm}, p\right|=\left(\left|0_{\mp}, p\right\rangle\right)^{\dagger},\left\langle 0_{+}, p \mid 0_{+}, p^{\prime}\right\rangle=0$, $\left\langle 0_{+}, p \mid 0_{-}, p^{\prime}\right\rangle=\delta^{10}\left(p+p^{\prime}\right)$, and the position space vacuum is defined by the standard Fourier transformation $\left|x_{0}\right\rangle=\int \frac{d^{10} p}{(2 \pi)^{5}}|p\rangle e^{i p x_{0}}$.

These boundary states break half of the 32 real supersymmetries of the type-IIB theory in ten dimensions. Writing the charges in the Green-Schwarz formulation as $Q^{a}$ and $Q^{\dot{a}}$, and similarly for world-sheet right-movers, we have $\frac{1}{\sqrt{2}}(Q \pm i \bar{Q})\left|I_{ \pm}, p\right\rangle=0$. Therefore, the ten-dimensional D-instantons have a total of 16 fermionic zero modes. Because of this BPS character, Dirichlet amplitudes with either instantons or anti-instantons, but not both, have unbroken supersymmetries that can be used to prove vanishing theorems by

4 As usual, the indices $i, a, \dot{a}$ run in the $\mathbf{8}_{\mathbf{v}}, \mathbf{8}_{\mathbf{s}}$ and $\mathbf{8}_{\mathbf{c}}$ of $S O(8)$, respectively. 
means of world-sheet Ward identities (see Section 3.2). This is not the case for amplitudes involving both instantons and anti-instantons, or for any amplitude at finite temperature, since the supersymmetry is then completely broken by the vacuum boundary conditions.

At finite temperature, the boundary states are exactly given by (2.4) in the oscillator sectors. At the level of the zero modes we simply have to implement the Kaluza-Klein quantization of the time-like momenta in the closed string channel $p^{0}=2 \pi n / \beta$, and the completeness relation for discrete thermal momenta becomes the usual $\langle n \mid m\rangle=\beta^{-1} \delta_{n, m}$. In the open string channel, as we shall see in more detail below, these discrete states are interpreted as winding modes of open strings around the thermal circle, which now carry a winding topological charge because their ends are constrained to lie at the instanton location.

The extreme dilute approximation is defined by dropping all interaction terms in (2.3) beyond the single-instanton effective action. For example, the contribution to the free energy or vacuum energy in a toroidal space $\mathbf{R}^{9} \times S_{\beta}^{1}$ is given by

$$
e^{-\beta F(\beta)}=e^{-\Gamma_{0}} \sum_{n_{+}=n_{-}} \frac{1}{n_{+} ! n_{-} !}\left(\int d \mu_{+} e^{-\Gamma_{1}^{+}}\right)^{n_{+}}\left(\int d \mu_{-} e^{-\Gamma_{1}^{-}}\right)^{n_{-}}
$$

where the constraint of neutrality $n_{+}=n_{-}$appears because the D-instantons are sources of $\mathrm{R}-\mathrm{R}$ flux, and charge neutrality is necessary for consistency in a compact space. Assuming $d \mu_{+}=d \mu_{-}$and writing the constraint as $\delta_{n_{+}, n_{-}}=\int_{0}^{2 \pi} \frac{d \theta}{2 \pi} e^{-i \theta\left(n_{+}-n_{-}\right)}$, we can exponentiate the result to

$$
e^{-\beta F(\beta)}=e^{-\Gamma_{0}} \int_{0}^{2 \pi} \frac{d \theta}{2 \pi} \exp \left(2 \int d \mu e^{-\Gamma_{1}} \cos \theta\right)
$$

If the measure $d \mu$ contains fermionic collective coordinates due to fermionic zero modes of the instantons, the non-perturbative corrections to the free energy in (2.6) clearly vanish. At finite temperature, however, all supersymmetries are broken by the vacuum boundary conditions (thermal antiperiodicity of fermions). We take the attitude that collective coordinates should be associated only to symmetries broken by the localized instanton, but restored asymptotically at infinity; otherwise we have a spontaneously broken symmetry and the collective modes are really Goldstone bosons. So, at finite temperature we expect the integral over the moduli to produce simply a factor of the ten-dimensional volume. 
The sum over vacuum diagrams $\Gamma_{0}$ is just the perturbative free energy, so we end up with a $\theta$-band

$$
F(\beta, \theta)_{\text {dilute }}=F(\beta)_{\text {pert }}-C \cdot \operatorname{Vol}_{9} \cdot e^{-\Gamma_{1}(\beta)} \cos \theta
$$

with $C$ some positive constant of order $\mathcal{O}(1)$ in string units. In the next section we will see that $\theta$ is just the zero mode of the dynamical axion field, so that we have to minimize (2.7) and relax to $\theta=0$. It is already clear from eq. (2.7) that hard singularities in the oneinstanton effective action $\Gamma_{1}(\beta)$ could in principle change the critical behaviour of the free energy. For example, a power behaviour at the Hagedorn temperature $\Gamma_{1}(\beta)_{\operatorname{sing}} \sim-\mid \beta-$ $\left.\beta_{H}\right|^{\alpha}$, with $\alpha \geq 0$, would turn the Hagedorn temperature into a maximum temperature, although a safer interpretation would be that the instanton expansion (in the sense of a weak-coupling expansion) breaks down badly, because a term nominally suppressed as $e^{-|Q| / \lambda}$ would dominate the physics.

The dilute approximation is constructed as a perturbative expansion around the gas of free instantons, according to the general expressions (2.1)-(2.3). The first corrections come from the 2-body interaction terms $\Gamma_{j, j^{\prime}}, \Gamma_{k, k^{\prime}}$ and $\Gamma_{j, k}$, which at low energies reduce to Coulomb interactions. We shall see in the next section that these terms generate periodic potentials for the carriers of these forces.

\subsection{Low-Energy Solutions}

At the massless level the D-instanton couples to the dilaton-graviton system and the $\mathrm{R}-\mathrm{R}$ axion field. It is therefore possible to describe the $\mathrm{D}$-instanton projection over the massless fields as a classical solution of the corresponding type-IIB supergravity theory. In this section we review some properties of the solution described in 13 and use it to study some dynamical aspects, which are most easily handled in the field theory framework, such as a derivation of an effective Lagrangian by integrating out the instantons in a dilute approximation.

In order to match the string coupling dependence with the previous stringy description

of the D-instanton gas, we need to use a metric frame which approaches the string frame at least asymptotically. At the same time we would like to have a space-time independent Newton constant by decoupling the dilaton from the trace of the gravitational metric. We can reconcile these two requirements by going to the "modified Einstein frame" [19], which is related to the string frame by the Weyl rescaling

$$
\left(g_{\mu \nu}\right)_{\mathrm{str}}=e^{\frac{\phi-\phi_{\infty}}{2}} g_{\mu \nu},
$$


which differs from the standard Einstein frame by a constant rescaling by the asymptotic value of the string coupling $\lambda=\lambda_{\infty} \equiv e^{\phi_{\infty}}$. In the following we will use this normalization of the metric, which leads to a slight change with respect to the string coupling conventions of [13].

Since the D-instanton is magnetically charged with respect to the $\mathrm{R}-\mathrm{R}$ 8-form potential of the type-IIB theory, it arises as a classical solution of the Euclidean action

$$
S=\frac{1}{2 \kappa_{0}^{2} \lambda^{2}} \int \sqrt{g}\left(-R+\frac{1}{2}(\partial \phi)^{2}+\frac{\lambda^{4}}{2} e^{-2 \phi} F_{9}^{2}+\ldots\right) .
$$

Here $\kappa_{0}^{2} \sim \alpha^{4}$ relates the string scale and the Newton constant 5 . For a single D-instanton located at the origin we have

$$
\begin{aligned}
d s_{c \ell}^{2} & =d r^{2}+r^{2} d \Omega_{9}^{2} \\
e^{\phi_{c \ell}} & =\lambda\left(1+\frac{\lambda}{8} \frac{|C|}{r^{8}}\right) \\
\left(F_{9}\right)_{c \ell} & =C d \Omega_{9},
\end{aligned}
$$

with $C$ a constant that will be determined shortly. The most important property of (2.10) is the total classical decoupling of gravity in the Einstein frame (or modified Einstein frame here), as the classical metric is flat. This fact makes the low-energy analysis of these instantons mostly independent of the more complicated gravitational sector.

The solution (2.10) is "self-dual" in the sense that

$$
d \phi_{c \ell}+\lambda^{2} e^{-\phi_{c \ell}} *\left(F_{9}\right)_{c \ell}=0
$$

which is in fact the BPS condition for preserving half of the total $N=2$ ten-dimensional type-IIB supersymmetry. As stated before, this property means that multi-instantons are easily constructed by direct superposition of the single-instanton solution in (2.10).

The local string coupling field $\lambda(x)=e^{\phi(x)}$ is harmonic in bulk, $\partial^{2} e^{\phi_{c \ell}}=0$, from which we derive the useful identity $\left(\partial \phi_{c \ell}\right)^{2}=-\partial^{2} \phi_{c \ell}$, which is valid away from the instanton location. Taken together with the BPS property (2.11), it allows a simple calculation of the instanton action with the result

$$
S_{c \ell}=\frac{1}{2 \kappa_{0}^{2} \lambda^{2}} \int\left(\frac{1}{2}\left(\partial \phi_{c \ell}\right)^{2}+\frac{\lambda^{4}}{2}\left(F_{9}\right)_{c \ell}^{2}\right)=\frac{1}{2 \kappa_{0}^{2} \lambda^{2}} \cdot \lambda\left|S^{9}\right||C|=\frac{|Q|}{\lambda}
$$

5 There is a natural normalization of this parameter in the type-IIB theory as the appropriate ratio of the D-string and fundamental string tensions, see [20]. 
with $\left|S^{9}\right|$ the volume of the unit nine-sphere and $|Q| \equiv W_{0}$ the disk amplitude. This fixes the constant $C= \pm \frac{2 \kappa_{0}^{2}|Q|}{\left|S^{9}\right|}$. The sign of $Q$ is defined as the sign of the flux

$$
\frac{1}{2 \kappa_{0}^{2}\left|S^{9}\right|} \oint_{\infty} F_{9}=Q
$$

and it is positive or negative according to the instanton or anti-instanton character of the solution.

We have seen that the instanton is a Coulomb "magnetic" source for $F_{9}$, much in the same way as 't Hooft-Polyakov monopoles arise as instantons in $2+1$ models. By Dirac duality, the D-instanton must be electrically charged with respect to the axion field dual to the 8-form. In the Euclidean formalism we must implement the Dirac duality by means of a Gaussian transformation of the $A_{8}$ path integral by the change of variables (see [21] for a general treatment of such manipulations)

$$
\left[\mathcal{D} A_{8}\right]_{\text {inst }} \rightarrow \mathcal{D} F_{9} \delta\left[d F_{9}-2 \kappa_{0}^{2} * J_{0}\right] \sim \int \mathcal{D} a \mathcal{D} F_{9} e^{-i \int a \wedge\left(\frac{1}{2 \kappa_{0}^{2}} F_{9}-* J_{0}\right)}
$$

which introduces the axion field as a Lagrange multiplier. In changing variables from $A_{8}$ to the field strength defined locally as $F_{9} \sim d A_{8}$, we have to exhibit explicitly the magnetic sources $J_{0}$, which prevent $F_{9}$ from being a closed form. Under this change of variables we can establish the duality by direct evaluation of the $F_{9}$ path integral, leading to

$$
\int\left[\mathcal{D} A_{8}\right]_{\text {inst }} e^{-\frac{\lambda^{2}}{4 \kappa_{0}^{2}} \int e^{-2 \phi}\left|F_{9}\right|^{2}} \sim \int \mathcal{D} a e^{-\frac{1}{4 \kappa_{0}^{2} \lambda^{2}} \int e^{2 \phi}|d a|^{2}-i \int a \wedge * J_{0}}
$$

and the saddle point in $F_{9}$ determines the Euclidean duality

$$
\left(F_{9}\right)_{\text {saddle }}=i \frac{e^{2 \phi}}{\lambda^{2}} * d a
$$

Using (2.16) we can infer the classical axion profile in the instanton background that would solve the axionic action

$$
S_{\text {axionic }}=\frac{1}{2 \kappa_{0}^{2} \lambda^{2}} \int \sqrt{g}\left(-R+\frac{1}{2}(\partial \phi)^{2}+\frac{1}{2} e^{2 \phi}(\partial a)^{2}\right)+\text { sources }
$$

and it is given by

$$
d a_{c \ell}=i e^{-\phi_{c \ell}} d \phi_{c \ell},
$$


from which we can integrate the axion field as

$$
a_{c \ell}-a_{\infty}=-i\left(e^{-\phi_{c \ell}}-\frac{1}{\lambda}\right) .
$$

Notice that a new integration constant appears, the asymptotic value of the axion at infinity, and that the local axion field is complex. As a result, the full instanton action comes from the source term, as the bulk contribution from (2.17) vanishes on account of the complex axion field. The normalization for the "current" of a D-instanton located at $x_{0}$ is adjusted as

$$
J_{0}=Q \delta^{10}\left(x-x_{0}\right)
$$

so that it leads to the correct statistical weight

$$
e^{-S_{c \ell}}=e^{-S_{\text {sources }}}=e^{-i \int a_{c \ell} \wedge * J_{0}}=e^{-i a_{c \ell}\left(x_{0}\right) Q}=e^{-\frac{|Q|}{\lambda}-i Q a_{\infty}}
$$

In view of the $S L(2, \mathbf{R})$ duality of type-IIB supergravity, the natural prescription is to take $a_{\infty}$ as a real number, which is in turn interpreted as a theta parameter, effectively compactified as an angular variable of period $2 \pi / Q$ when the non-perturbative dynamics breaks the continuous duality symmetry to $S L(2, \mathbf{Z})$.

Finite-temperature solutions are straightforward in terms of the single-instanton solution (2.10). Because of the BPS property we are free to superimpose multi-instantons, and a periodic configuration in Euclidean time is easily obtained by summing all discrete translations of period $\beta$ in the time direction. For example, we have for the dilaton profile

$$
e^{\phi_{c \ell}}=\lambda+\frac{\lambda^{2}}{8} \sum_{n \in \mathbf{Z}} \frac{|C|}{\left|x-x_{0}+n \beta e^{0}\right|^{8}},
$$

with $e^{0}$ a unit vector in the time direction. Now the action has to be integrated only over the cylinder $0<x<\beta$ in the time direction, and the result is easily checked to be the same as in the vacuum case $S_{c \ell}=|Q| / \lambda+i a_{\infty} Q$. In fact, when computed in the axionic form (2.17) and (2.21), the result is trivial, because it comes from a delta-function-localized source term. This is compatible with the full stringy determination, where the leading classical action comes from the disk diagram. Since the disk boundary is mapped to the instanton location, no winding modes are allowed and no temperature dependence can appear to this order. We also see that $a_{\infty} Q$ is nothing but the theta parameter introduced in (2.6) to enforce charge neutrality in the presence of compact directions, and therefore $a_{\infty}$ is to be integrated between 0 and $2 \pi / Q$. 


\subsection{Instanton Measure}

The instanton measure is generated by quantum corrections to the classical solution, and it naturally splits into two qualitatively different contributions: zero modes, inducing the collective coordinates measure $d \mu$, and perturbative quantum corrections due to nonzero modes, which are summarized in the non-classical part of the one-instanton effective action

$$
\Gamma_{1} \text { (quantum) }=\sum_{g>0} \sum_{N>0} \frac{\lambda^{2 g+N-2}}{N !} W_{g, N}
$$

which contains string diagrams with at least one loop of closed strings, and at least one instanton insertion via a Dirichlet boundary. On the other hand, the "classical" action is given by the genus-zero terms

$$
\Gamma_{1}(\text { classical })=S_{c \ell}=\frac{W_{0,1}}{\lambda}+\sum_{N>1} \frac{\lambda^{N-2}}{N !} W_{0, N}
$$

In type-IIB strings, space-time supersymmetry together with the BPS character of the D-instantons implies that $W_{0, N}=0$ for $N>1$ and therefore there are no perturbative corrections to the disk contribution to the classical action. This is not the case for purely bosonic D-instantons.

The full one-loop measure is given by

$$
d \mu A_{1-\mathrm{loop}}=d \mu \exp \left(-\sum_{N>0} \frac{\lambda^{N}}{N !} W_{1, N}\right)
$$

in the full string theory, which should go over the standard determinant factor in the low-energy $\alpha^{\prime} \rightarrow 0$ limit

$$
A_{1-\text { loop }}=1-\lambda W_{1,1}+\mathcal{O}\left(\lambda^{2}\right) \rightarrow\left(\frac{\operatorname{det}^{\prime} S^{(2)}(\text { inst })_{\text {g.f. }}}{\operatorname{det}^{\prime} S^{(2)}(\mathrm{vac})_{\text {g.f. }}}\right)^{-\frac{1}{2}}\left(\frac{\operatorname{det}^{\prime} D(\text { inst })}{\operatorname{det}^{\prime} D(\operatorname{vac})}\right) .
$$

Here $S_{\text {g.f. }}^{(2)}$ stands for the bosonic fluctuation kernel of the low-energy theory with possible gauge-fixing (ghost) components, and $D$ is the corresponding fermionic kernel. There are a few subtleties in the limit (2.26): each determinant factor in the right-hand side contains an infinite series of powers of the string coupling. In the stringy construction, the states running in the propagators in (2.23) are just free string states in flat space; therefore the real loop-counting parameter of the low-energy theory is just the number of closed 
string loops. If we would rearrange (2.23) into string field theory diagrams and take the low-energy limit we would find for example the fermionic determinant ratio in the form

$$
\sum_{n>0} \frac{(-\lambda)^{n}}{n}\left(\frac{1}{\gamma \cdot \partial} \varphi_{c \ell}\right)^{n}=-\operatorname{Tr} \log \left(1+\frac{1}{\gamma \cdot \partial} \lambda \varphi_{c \ell}\right)
$$

where $\varphi_{c \ell}$ represents the massless bosonic profiles of the instanton coupling to fermion bilinears in the low-energy effective theory. In the full string theory $\varphi_{c \ell}$ is associated to the tadpole $\left\langle V_{\varphi} \mid I\right\rangle$ in the D-instanton background. In fact, the existence of non-vanishing tadpoles more or less implies that this is the right interpretation of the low-energy limit. Notice also that, in the low-energy description, the instanton fields are not quantitatively significant beyond a string-scale "core", and therefore we may perturbatively expand the profile function. Since all profiles are controlled by the dilaton profile, we have an effective expansion in powers of the local string coupling. This is explicitly seen in the form of the only non-trivial one-loop kernel, from the axion-dilaton sector:

$$
\mathcal{K}_{\phi, a}=\left(\begin{array}{cc}
-\partial^{2}-2\left(\partial \phi_{c \ell}\right)^{2} & 2 i \partial_{\mu}\left(e^{\phi_{c \ell}}\right) \partial^{\mu} \\
-2 i \partial_{\mu}\left(e^{\phi_{c \ell}}\right) \partial^{\mu} & -\partial_{\mu} e^{2 \phi_{c \ell}} \partial^{\mu}
\end{array}\right)
$$

In practice, it is somewhat complicated to write explicit expressions for the right-hand side of (2.26), due to the presence of self-dual four-forms in the type-IIB theory, but there is no problem in giving a formal definition of the stringy expression (2.23). We will not need an explicit expression for the non-zero mode quantum effective action, which we denote by $A$ in the remainder of the paper. We just point out the general structure $A \sim 1+\mathcal{O}(\lambda)$, which is rather obvious in the stringy construction of $\Gamma_{1}$, as in (2.23).

This simple situation must be modified when quasi-zero modes appear for some values of the parameters. A characteristic example is the low-temperature limit $\beta \rightarrow \infty$ of the fermionic determinants, which develop a fermionic zero mode as supersymmetry is restored in the zero-temperature limit. Such quasi-zero modes must be subtracted and treated in terms of collective coordinates.

In (2.1), the collective coordinate measure $d \mu^{ \pm}$was not specified. Dirichlet instantons are distinguished from familiar Yang-Mills instantons in that they do not have scale moduli, which just conforms with the lack of scale invariance of the supergravity theory. In the full stringy description it is also clear that the only data specified by the boundary state (2.4) concern the space-time position of the instanton $x_{0}$. Then, we have just ten bosonic collective coordinates for the position, and a number of fermion collective coordinates 
associated to the zero modes generated by broken supersymmetries; sixteen of them in the full type-IIB theory. We will discuss here just the bosonic collective Jacobian, because it is the only one relevant at high temperatures, postponing to Appendix A a review of D-instanton fermionic collective coordinates, with emphasis on the treatment of the lowtemperature quasi-zero modes.

We can easily fix the leading term of the collective coordinate measure in the lowenergy supergravity theory. Since the low-energy solution (2.10) in the Einstein frame is only non-trivial along the axion-dilaton components in field space, we consider the zero-modes of the corresponding fluctuation operator $\mathcal{K}_{\phi, a}$ written in (2.28). The relevant quadratic form is

$$
S=S_{c \ell}+\frac{1}{2}\left\langle\left(\phi_{q}, a_{q}\right)|\mathcal{K}|\left(\phi_{q}, a_{q}\right)\right\rangle+\ldots
$$

The inner product defining the path integral measure for the quantum fluctuations $\phi_{q}, a_{q}$ is given by

$$
\left\langle\left(\phi_{q}, a_{q}\right) \mid\left(\phi_{q}^{\prime}, a_{q}^{\prime}\right)\right\rangle=\int d^{10} \xi\left(\frac{1}{\lambda^{2}} \phi_{q}^{*}(\xi) \phi_{q}^{\prime}(\xi)+a_{q}^{*}(\xi) a_{q}^{\prime}(\xi)\right)
$$

and we have rescaled $x=\left(2 \kappa_{0}^{2}\right)^{4 / 5} \xi$ to dimensionless variables. Then, a set of finite-norm zero-modes of $\mathcal{K}$ is given by

$$
V_{\alpha}=\left(\frac{\partial \phi_{c \ell}}{\partial \xi^{\alpha}}, \frac{\partial a_{c \ell}}{\partial \xi^{\alpha}}\right)
$$

and the resulting measure is calculated following the standard algorithm in [14]:

$$
d \mu=\prod_{\alpha=1}^{10} d \xi_{\alpha}\left(\frac{\left\langle V_{\alpha} \mid V_{\alpha}\right\rangle}{2 \pi}\right)^{\frac{1}{2}}
$$

Using the classical solutions, we easily calculate the Jacobian. By symmetry all terms in the product in (2.31) are equal, and therefore

$$
\left\langle V_{\alpha} \mid V_{\alpha}\right\rangle=\frac{1}{10} \sum_{\sigma=1}^{10}\left\langle V_{\sigma} \mid V_{\sigma}\right\rangle=\frac{1}{10} \int \frac{d^{10} x}{2 \kappa_{0}^{2}}\left(\frac{1}{\lambda^{2}}\left(\partial \phi_{c \ell}\right)^{2}-\left(\partial a_{c \ell}\right)^{2}\right)=\frac{1}{10}\left(1+\frac{1}{3}\right) \frac{|Q|}{\lambda}
$$

where we have used the fact that $\partial a_{c \ell}$ is purely imaginary and we obtain the final result

$$
d \mu_{\text {bosonic }} \equiv d^{10} x_{0} J_{\text {coll }}=\frac{d^{10} x_{0}}{\left(2 \kappa_{0}^{2}\right)^{5 / 4}}\left(\frac{|Q|}{15 \pi \lambda}\right)^{5}
$$

Notice the occurrence of the usual factor $\left(\sqrt{S_{c \ell}}\right)^{\nu}$ for $\nu$ translational zero modes. 
The integral over position collective coordinates must be supplemented with certain constraints. This is due to the existence of short-distance singularities in the perturbative setting described so far. Strictly speaking, only pure multi-instanton configurations are exact solutions but, as is well known in the field theory case, clustering implies that we must consider approximate solutions with well separated instantons and anti-instantons in order to have a consistent approximation. Short-distance singularities come from the corners of the approximate multi-instanton moduli space, corresponding to the coincidence limit of two or more instantons or anti-instantons 6 . For bosonic D-instantons, both types of degeneration lead to singularities. For type-IIB D-instantons, the BPS property implies regularity of the pure instanton (I) or pure anti-instanton (A) degenerations. However, the I-A degeneration is singular already at the classical level, since the cylinder diagram gives a divergence at half the Hagedorn distance $\beta_{H}=2 \pi \sqrt{2 \alpha^{\prime}}$ of the form $\Gamma_{I-A} \sim$ $-C \log \left|\Delta x-\frac{\beta_{H}}{2}\right|$, where $\Delta x$ is the I-A separation [11], [18], [23]. At zero temperature, fermion zero modes increase the constant $C$, thus enhancing the singularity [24].

The ambiguities in the I-A parametrization are well known already at the field theory level, since these are not topologically distinct configurations, and I-A pairs should annihilate into perturbative states. The sharp singularity that appears in the stringy case is one of the most interesting and pressing problems of D-instanton dynamics, and we will not discuss the question further in this paper. In the spirit of the dilute approach, we will assume that a "hard core" interaction exists around all D-(anti-) instantons, in such a way that the integral over their positions $x_{i}$ will be restricted to $\left|x_{i}-x_{j}\right|>\beta_{H} / 2$.

Actually, the hard core is needed even in the I-I sectors. The reason is that, as $N$ D-instantons come together at sub-stringy distances, there are $N^{2}$ light modes becoming massless that must be treated as quasi-zero modes and included into the collective coordinates [25], [26]. Therefore, the collective dynamics beyond the dilute approximation involves $U(N)$ matrix dynamics in the I-I sectors, and some unknown physics determining the I-A annihilation. For the applications discussed in the present paper (general structure of singularities in the single-instanton sector and the infrared analysis of the phase transition) we believe the dilute approximation to be appropriate. The limitations of our treatment should, however, be kept in mind.

6 At the classical level, the solution (2.10) develops local strong coupling at a distance of the order of the string scale, $\ell_{s} \sim \sqrt{\alpha^{\prime}}$, when measured in the weakly-coupled, dual type-IIB background [22]. 


\subsection{Instanton Interactions and Effective Lagrangians}

In analysing the physical effects of instantons in the dilute approximation, it is common practice to summarize the short-distance effects induced by instantons in an effective Lagrangian, which we can imagine as the result of integrating out the instanton fluctuations. This approach is specially appropriate for the D-instantons, since these have a fixed string-scale effective size. In general, one just considers [17] the corresponding amputated Green functions in the instanton background, and reinterprets them in the local limit as inducing a set of local operators

$$
\left\langle\prod_{\text {bosons }}\left(-\partial^{2}\right) \Phi \prod_{\text {fermions }}(\gamma \cdot \partial) \Psi\right\rangle \rightarrow \sum_{\text {local ops. }}\left\langle\mathcal{O}_{\text {eff }}(\Phi, \Psi)\right\rangle_{\text {vac }}
$$

For example, in the ten-dimensional type-IIB theory, D-instantons have sixteen fermionic zero modes, which have to be saturated in order to have a non-vanishing expectation value in the one-instanton sector. Therefore, to leading order, we induce operators with $n_{f}$ fermions, $n_{b}$ bosons and a number $n_{\partial}$ of derivatives of the form [24]

$$
\mathcal{O}_{\text {IIB }} \sim J_{\text {coll }} A e^{-|Q| / \lambda} \Psi^{n_{f}} \partial^{n_{\partial}} \Phi^{n_{b}}
$$

with $n_{\partial}+n_{f} / 2=8$. These are high-dimension operators, which are not very relevant in the infrared. However, in the situation without fermion zero modes given by the finite-temperature boundary conditions, purely bosonic lower-dimension operators can be generated. For example, the axion gets a mass from the one-instanton sector of the form

$$
m_{a}^{2}=J_{\text {coll }} A e^{-|Q| / \lambda}\left|\int \partial^{2} a_{c \ell}\right|^{2} \sim Q^{2} A J_{\text {coll }} e^{-|Q| / \lambda} .
$$

This formula comes from the single-instanton contribution to the two-point function $\left\langle a^{*}(x) a(y)\right\rangle_{\text {inst }}$ after we amputate external propagators and take the local limit. In order to interpret formula (2.37) we still need to argue that the axion effective potential attains its minimun at $a=0$. This is easily done in this problem, because including the two-body interactions modulates the mass terms as in (2.37) into periodic potentials, the reason being that the two-body interactions are Coulombic at long distances. Factorizing the massless exchange channel, we find no interactions between like-instantons at tree-level,

7 Recently, a careful analysis of an induced bosonic operator with 8 derivatives, an $R^{4}$ term, has appeared in [27]. 
due to the cancellation between the dilaton and axion exchanges, and a doubled attractive channel between instantons and anti-instantons,

$$
\begin{aligned}
\Gamma_{+, \pm}(x-y) & \sim \Delta(x-y)[\langle+| \text { axion }\rangle\langle\text { axion } \mid \pm\rangle-\langle+| \text { dilaton }\rangle\langle\text { dilaton } \mid \pm\rangle] \\
& =\Delta(x-y)\left( \pm Q^{2}-Q^{2}\right)
\end{aligned}
$$

where $\Delta(x-y)=\left\langle x\left|\left(-\partial^{-2}\right)\right| y\right\rangle$ is the Green function of the ten-dimensional Laplacian. We see that, even with the Coulomb interactions involved, we do not really have a plasma in the sense that there are no repulsions, and attractions are doubled with respect to the standard case. The corresponding partition function up to two-body interaction terms can be written as

$$
\mathcal{Z}_{2-\text { body }}=\sum_{n_{+}=n_{-} \geq 0} \int \frac{\left(d x^{+}\right)\left(d x^{-}\right)}{n_{+} ! n_{-} !}\left(A J_{\text {coll }} e^{-S_{c \ell}^{+}}\right)^{n_{+}}\left(A J_{\text {coll }} e^{-S_{c \ell}^{-}}\right)^{n_{-}} e^{-V_{\mathrm{int}}^{\phi}-V_{\mathrm{int}}^{a}}
$$

where $S_{c \ell}^{ \pm}= \pm i|Q| a_{\infty}+|Q| / \lambda$ and charge neutrality is enforced by integrating $a_{\infty}$ between 0 and $2 \pi / Q$. The Coulomb interaction potentials due to axion and dilaton exchange are given by a sum over $\mathrm{I}-\mathrm{I}, \mathrm{A}-\mathrm{A}$ and $\mathrm{I}-\mathrm{A}$ pairs (we set $2 \kappa_{0}^{2}=1$ for simplicity throughout this section):

$$
\begin{aligned}
& V_{\mathrm{int}}^{\phi}=-Q^{2} \sum_{\left(j, j^{\prime}\right)}^{n_{+}} \Delta_{j j^{\prime}}-Q^{2} \sum_{\left(k, k^{\prime}\right)}^{n_{-}} \Delta_{k k^{\prime}}-Q^{2} \sum_{(j, k)} \Delta_{j k} \\
& V_{\mathrm{int}}^{a}=+Q^{2} \sum_{\left(j, j^{\prime}\right)}^{n_{+}} \Delta_{j j^{\prime}}+Q^{2} \sum_{\left(k, k^{\prime}\right)}^{n_{-}} \Delta_{k k^{\prime}}-Q^{2} \sum_{(j k)} \Delta_{j k}
\end{aligned}
$$

where we have suppressed the self-energy terms because they cancel in the sum $V^{\phi}+V^{a}$. By means of a usual Gaussian trick we can express the interactions in terms of path integrals for non-zero modes of the axion and dilaton fields $\mathrm{B}$

$$
\begin{aligned}
& e^{-V_{\mathrm{int}}^{\phi}}=\operatorname{det}^{1 / 2}\left(-\partial^{2}\right) \int \mathcal{D} a^{\prime} e^{-\frac{1}{2} \int d x\left[\left(\partial a^{\prime}\right)^{2}-i a^{\prime}\left(J_{0}^{+}-J_{0}^{-}\right)\right]} \\
& e^{-V_{\mathrm{int}}^{a}}=\operatorname{det}^{1 / 2}\left(-\partial^{2}\right) \int \mathcal{D} \phi^{\prime} e^{-\frac{1}{2 \lambda^{2}} \int d x\left[\left(\partial \phi^{\prime}\right)^{2}+\lambda \phi^{\prime}\left(J_{0}^{+}+J_{0}^{-}\right)\right]}
\end{aligned}
$$

with $J_{0}^{ \pm}= \pm|Q| \sum_{i=1}^{n_{ \pm}} \delta^{10}\left(x-x_{0}^{ \pm}\right)$the instanton or anti-instanton currents as defined in (2.20). Now substituting back in (2.39) and summing the series, we arrive at the effective

8 That is, the path integral measure is taken in the functional space orthogonal to the zero modes of the Laplacian $-\partial^{2}$. 
ten-dimensional Euclidean Lagrangian (here we benefit from using the modified Einstein frame):

$$
\mathcal{L}_{\mathrm{eff}}=\frac{1}{2 \lambda^{2}}\left(\partial \phi^{\prime}\right)^{2}+\frac{1}{2}\left(\partial a^{\prime}\right)^{2}-2 A J_{\mathrm{coll}} e^{-|Q| / \lambda} e^{-\frac{|Q|}{\lambda} \phi^{\prime}} \cos \left[Q\left(a^{\prime}+a_{\infty}\right)\right]
$$

which we can understand as the Gaussian approximation to an effective Lagrangian for the full local string coupling $\log \lambda(x)=\phi(x) \sim \log \lambda+\phi^{\prime}(x)+\mathcal{O}\left(\phi^{2}\right)$ and the full axion field $a=a^{\prime}+a_{\infty}$ with an effective potential 9

$$
V_{\text {eff }}=-2 A J_{\text {coll }} e^{-|Q| / \lambda(x)} \cos (Q a)
$$

So, we find the standard result that, when allowed by zero-mode counting, instantons generate a periodic potential for the axion, but also a runaway potential towards the trivial free theory for the dilaton, a well-known pathology of weakly coupled string theory.

At finite temperature, the effective ten-dimensional Lagrangian described here only makes sense for $\beta \gg \sqrt{\alpha^{\prime}}$ because we are assuming a hard core of stringy size. In the discussion of the last section we will be interested in the critical behaviour at temperatures of the order of the string scale. An effective Lagrangian approach is still useful if we concentrate on the long wave-length dynamics in the spatial directions.

An important point regarding $(2.43)$ is the physics it contains. The only corrections to the free-instanton limit are given by the tree-level Coulomb interaction, corresponding to the cylinder diagram $W_{0,2}$ in the full string theory. In field theory, instanton interactions induced by one-loop diagrams in the multi-instanton terms come from diagrams with at least one handle and two boundaries, and are therefore suppressed by extra powers of the string coupling. Thus, (2.43) is a consistent approximation within the weak coupling expansion.

9 Notice that we integrate the axion zero mode, which is natural in a compact space. However, we do not integrate over the dilaton zero mode, which determines the asymptotic string coupling and poses a real super-selection rule. This is reminiscent of similar issues in Liouville field theory, and is characteristic of runaway potentials [28]. 


\section{Singularities of the Free Energy}

In perturbation theory both the canonical free energy and the internal energy of a gas of ten-dimensional type-IIB superstrings are finite quantities 10 at the Hagedorn temperature $T_{H}$. This is generally interpreted [29] in the sense that the string collective undergoes some kind of phase transition near $T_{H}$, after which strings may no longer be the most adequate degrees of freedom to describe the physics at high temperatures [30].

One legitimate question is whether or not the kind of non-perturbative effects represented by D-instantons can change in any way the critical behaviour of the type-IIB superstring at the Hagedorn temperature. This may not seem to be very likely at first sight, since D-instanton effects are exponentially suppressed with respect to the perturbative quantum corrections at weak coupling. On the other hand one must remember that D-instantons are related to open strings by T-duality and that for open strings the Hagedorn temperature is a maximum temperature of the Universe where the internal energy diverges. Therefore there could be a scenario in which the non-perturbative contribution would drive the internal energy to infinity when approaching the Hagedorn temperature from below.

In general, singularities will appear in the free energy or its derivatives with respect to the moduli fields whenever there are extra massless states emerging at some point of the moduli space. This is also true in the thermal case, where the only modulus now is the temperature $T$, but the situation here is even worse since the state becoming massless at the Hagedorn temperature turns out to be tachyonic above $T_{H}$, rendering the whole perturbative expansion meaningless. The squared effective mass of this state generically scales with the inverse temperature $\beta$ as $\alpha^{\prime} m_{\text {eff }}^{2} \sim\left(\beta^{2} / \alpha^{\prime}\right)-a$, where $a>0$; therefore the state triggering the Hagedorn transition corresponds to a winding string. For the closed string models this winding mode comes from the usual solitonic sector wrapping around the compactified Euclidean time. The picture is different in the presence of a D-instanton located at $x_{0}$ since we then have open strings whose endpoints are stuck at this position, and this produces topological sectors of a new kind, corresponding to winding open strings. It is easy to see that these are the states producing new thermal singularities in instanton diagrams.

10 For the closed bosonic string this is also true once we remove the divergence associated with the ground-state tachyon. 
To be more explicit, we will illustrate the relevant points in the context of bosonic strings and D-instantons, whose boundary states are easily obtained by dropping the fermionic dependence in (2.4). Because of the zero-temperature tachyon instability of the bosonic string, the present discussion is only illustrative and an explicit disentangling of the singularities between thermal and non-thermal is required. Later, we will address the case of the type-IIB superstring where the absence of a zero-temperature tachyon makes the discussion more interesting from the physical point of view.

The first temperature-dependent contribution to the free energy comes from the sphere with two boundaries attached to the D-instanton, i.e. the annulus with Dirichlet boundary conditions:

$$
W_{0,2}(\beta)=-\frac{\pi^{12}\left(2 \pi \alpha^{\prime}\right)^{13}}{2} \int_{0}^{\infty} d s\left\langle I, x_{0}\left|e^{-s \Delta_{\text {closed }}}\right| I, x_{0}\right\rangle=-\frac{1}{2} \int_{0}^{\infty} \frac{d t}{t} \operatorname{Tr} e^{-t \Delta_{\text {open }}^{D}}
$$

where we have exhibited the modular transformation $t=2 \pi^{2} / s$ interchanging the open and closed string channels, and the normalization is determined by the open string channel. In the closed channel we have tree-level propagation of closed strings between Dirichlet boundary states with world-sheet Hamiltonian $\Delta_{\text {closed }}=\frac{\alpha^{\prime}}{2} \vec{p}^{2}+\frac{2 \pi^{2} \alpha^{\prime} n^{2}}{\beta^{2}}-2+$ osc. Notice that only momentum modes of closed strings propagate in this channel: the closed strings cannot wind around the thermal circle because they are mapped into the instanton location. Discarding the zero-temperature divergences, the first thermal singularity appears at the $s \sim 0$ endpoint, which is therefore better parametrized in the modular transformed open channel. In the open channel we have a one-loop vacuum amplitude of open strings whose endpoints are completely fixed at the instanton position (i.e. they have no momentum modes), but are allowed to wind a number of times around the thermal circle. The corresponding world-sheet Hamiltonian is

$$
\Delta_{\text {open }}^{D}=\frac{\beta^{2} n^{2}}{4 \pi^{2} \alpha^{\prime}}-1+\text { oscillators. }
$$

Notice the balance between the zero-point Casimir energy responsible for the open-string tachyon, and the positive stretching energy of the open string with winding number $n$. In this parametrization, the amplitude (3.1) takes the form $\left(w=e^{-t}\right.$, and $f(w)=\prod_{m>0}(1-$ $\left.\left.w^{m}\right)\right)$ :

$$
W_{0,2}(\beta)=-\frac{1}{2} \int_{0}^{\infty} \frac{d t}{t} \sum_{n \in \mathbf{Z}} \frac{e^{-t \Delta_{D, \text { open }}^{(0)}}}{f(w)^{24}}=-\frac{1}{2} \int_{0}^{\infty} \frac{d t}{t} \sum_{n \in \mathbf{Z}} \frac{e^{-t\left(\frac{\beta^{2} n^{2}}{4 \pi^{2} \alpha^{\prime}}-1\right)}}{f(w)^{24}}
$$


From here we see that, modulo the zero-temperature tachyon instability, $W_{0,2}(\beta)$ diverges logarithmically when $\beta$ approaches $\beta_{c}=2 \pi \sqrt{\alpha^{\prime}}$ and is divergent when $\beta<\beta_{c}$. After an analytic continuation we find that, close to the critical inverse temperature,

$$
W_{0,2}(\beta) \rightarrow \log \left(\beta-\beta_{c}\right)+\text { subleading. }
$$

Here $\beta_{c}$ corresponds to the self-dual length under T-duality and since $\beta_{c}=\beta_{H} / 2<\beta_{H}$, it lies well inside the region of values of $\beta$ for which the perturbative contribution is already divergent. From (3.3) we can read the effective masses of the states and check that the divergence at $\beta_{H} / 2$ is produced by a state with winding number equal to 1 and oscillator number equal to 0 . As a matter of fact we have a whole collection of critical temperatures at $\beta_{n}=\beta_{H} / 2 n$ triggered by the tachyonic ground state wrapping $n$ times around the compactified Euclidean time.

For type-IIB strings, possible tachyonic winding modes of open strings would appear in the Neveu-Schwarz sector, which has a negative Casimir term. The corresponding worldsheet Hamiltonian would be $\Delta_{\text {open }}^{D}(N S)=\frac{\beta^{2} n^{2}}{4 \pi^{2} \alpha^{\prime}}-\frac{1}{2}+$ osc, and the critical point lies again at $\beta_{c}=\pi \sqrt{2 \alpha^{\prime}}$, twice the Hagedorn temperature of type-IIB strings. We conclude that, within the canonical ensemble, the single D-instanton sector gives smooth contributions to the free energy at the Hagedorn point; new singularities, although they are hard, lie well beyond the range of applicability of the perturbative D-instanton construction.

\subsection{D-instanton Singularities and the Microcanonical Ensemble}

At face value, the fact that the singularities of $W_{2,0}(\beta)$ appear inside the Hagedorn domain seems to indicate that they have no physical consequence for the thermodynamics of the string gas. As we will see immediately, the fundamental clue to realize that this does not have to be the case lies in using the microcanonical ensemble [3], [32], [33], [34] 11 .

In the microcanonical description, the fundamental object is the density of states $\Omega(E)=\sum_{a} \delta\left(E-E_{a}\right)$, where the sum runs over all states in the system. All thermodynamical quantities are obtained from derivatives of the entropy $S=\log \Omega(E)$; for example

11 We thank M.A.R. Osorio for pointing out the possible relevance of the microcanonical ensemble. 
the microcanonical temperature $T$ and the specific heat at constant volume $c_{V}$ are given by

$$
T=\left(\frac{\partial S}{\partial E}\right)^{-1}, \quad c_{V}=-\frac{1}{T^{2}}\left(\frac{\partial^{2} S}{\partial E^{2}}\right)^{-1} .
$$

Needless to say, a direct exact computation of the microcanonical density of states in string theory is a desperate task. On the other hand the canonical free energy, and thus the partition function, can be easily computed at least at one loop just by knowing the spectrum of the theory [35] [36]. It is well known that there is a simple relation between the two quantities; by using an integral representation of the delta function in the definition of $\Omega(E)$, it is possible to rewrite it in terms of the analytic continuation of the canonical partition function $\mathcal{Z}(\beta)=\exp (-\beta F(\beta))$ by the complex inversion formula

$$
\Omega(E)=\int_{c-i \infty}^{c+i \infty} \frac{d \beta}{2 \pi i} \mathcal{Z}(\beta) e^{\beta E}
$$

where $c \in \mathbf{R}$ and is such that $c>\operatorname{Re} \beta_{i}$ for all the singular points $\beta_{i}$ of $\mathcal{Z}(\beta)$.

Although (3.5) provides us with a simple way to obtain $\Omega(E)$ from known quantities, an actual exact computation of the integral seems to be out of the question. Instead, the authors of ref. [34] designed a method to get the terms of the asymptotic expansion of $\Omega(E)$ for large $E$; by pushing $c$ to the left in the complex plane and deforming the contour to avoid the singular points, one finds that the leading contributions to (3.5) come from the integration around the singularities of $\mathcal{Z}(\beta)$; the further to the left we place $c$ the more singular points we have to integrate around and the more terms we get in the asymptotic expansion. So the lesson to be learned is that all the singularities of $\mathcal{Z}(\beta)$ in the complex $\beta$ plane contribute to $\Omega(E)$, even those lying to the left of $\beta_{H}$. In Appendix B we have outlined the calculation to obtain the perturbative contribution to $\Omega(E)$ at one loop for the bosonic string, using the procedure just described.

On general grounds, a singularity $\mathcal{Z}(\beta) \sim\left(\beta-\beta_{c}\right)^{\alpha}$ leads, after an inverse Laplace transform, to a contribution to the asymptotic density of states of the form $E^{-(1+\alpha)} e^{\beta_{c} E}$ (see Appendix B). A consequence of this is that the leading behaviour of $\Omega(E)$ in the large energy regime associated with each singular temperature will be given by the lowest critical exponents of $\mathcal{Z}(\beta)$. Since all the singularities have an infrared interpretation, the corresponding critical exponents can be easily extracted from the low-energy effective theory of light modes in $S_{\beta}^{1} \times \mathbf{R}^{d}$. The strength of the infrared singularity is characterized by the "effective dimension" $d_{\text {eff }}$, the number of spatial dimensions that the centre of mass 
of the string propagating in the loop is able to probe. Morally speaking, for a standard (open or closed) propagating string state with $m_{\text {eff }}^{2} \sim \beta-\beta_{c}$ we have at one loop

$$
F(\beta)_{1-\text { loop }} \sim \int_{0}^{\infty} \frac{d s}{s} \int d^{d_{\text {eff }}} \vec{p} e^{-s\left(\vec{p}^{2}+\beta-\beta_{c}\right)} \sim\left(\beta-\beta_{c}\right)^{d_{\text {eff }} / 2} .
$$

Actually, this case is special because of the extra $1 / s$ in the moduli measure. At higher order $(2 g+N \geq 2)$ we must estimate infrared divergences in propagators rather than in the logarithm of a determinant 12 . From the corner of the moduli space with $n$ soft loops of the same type, $n \leq g$ or $N-1$, we get a contribution

$$
F(\beta)_{n} \sim \lambda^{2 g-2}\left[\int_{0}^{\infty} d s \int d^{d_{\text {eff }}} \vec{p} e^{-s\left(\vec{p}^{2}+\beta-\beta_{c}\right)}\right]^{n} \sim \lambda^{2 g-2}\left(\beta-\beta_{c}\right)^{n\left(d_{\text {eff }} / 2-1\right)} .
$$

As we saw, in the large- $E$ limit the leading term comes from the smallest critical exponent. From (3.7) we see that we will run into trouble whenever $d_{\mathrm{eff}} \leq 2$, because then an instability takes over in the sense that the singularity gets harder as we increase the order of the diagram. This would indicate that the weak-coupling expansion breaks down at high energies, since higher-order Riemann surfaces would dominate the large energy limit. As we will see later, this is exactly what happens in the one-instanton sector of the bosonic string, where $d_{\text {eff }}=0$ for "soft strips" (the open strings endpoints are stuck at the instanton location). More generally, if we are doing thermodynamics in the background of a fixed $p$-brane, we will find problems for $p=0,1,2$.

On the other hand, for closed string soft loops, $d_{\mathrm{eff}}=d>2$. In the perturbative (zero-instanton) sector, these are the only types of handles present and therefore, for higher genus surfaces, the leading term in $\Omega(E)$ comes from a Riemann surface with a single "soft handle". Incidentally, the effective dimension for the interaction contributions $(g>1)$ is two units smaller than the one for $g=1$, so these diagrams could dominate the torus contribution if the energy is high enough, in spite of their suppression by powers of the (small) string coupling.

As is usual in instanton calculus, all physical quantities have to be expressed in a weak-coupling expansion around each instanton sector. This entails the following form for the partition function

$$
\mathcal{Z}(\beta)=\sum_{n=0}^{\infty} \lambda^{2 n} \mathcal{Z}_{2 n}(\beta)+e^{-|Q| / \lambda} \sum_{m=0}^{\infty} \lambda^{m} \mathcal{Z}_{m}^{\prime}(\beta)+\mathcal{O}\left(e^{-2|Q| / \lambda}\right) .
$$

12 This can be seen alternatively as due to the fact that there are no residual Killing vectors associated with higher-order Riemann surfaces. 
Using the linear relation (3.5) between the partition function and the density of states we obtain the representation for $\Omega(E)$ in the dilute-gas approximation:

$$
\Omega(E)=\sum_{n=0}^{\infty} \lambda^{2 n} \Omega_{n}(E)+e^{-|Q| / \lambda} \sum_{m=0}^{\infty} \lambda^{m} \Omega_{m}^{\prime}(E)+\mathcal{O}\left(e^{-2|Q| / \lambda}\right) .
$$

Then, the contributions of the one-D-instanton sector to $\Omega(E)$ in eq. (3.8) are defined by

$$
\Omega_{m}^{\prime}(E)=\int_{c-i \infty}^{c+i \infty} \frac{d \beta}{2 \pi i} \mathcal{Z}_{m}^{\prime}(\beta) e^{\beta E}=\int_{c-i \infty}^{c+i \infty} \frac{d \beta}{2 \pi i}\left[e^{-\beta F(\beta)_{\mathrm{pert}}-\Gamma_{1}^{\prime}(\beta)}\right]_{m} e^{\beta E}
$$

where the subscript $m$ indicates that we are only retaining the coefficient multiplying $\lambda^{m} e^{-|Q| / \lambda}$ in the expansion in powers of $\lambda$, and

$$
\Gamma_{1}^{\prime}(\beta)=\sum_{N=1}^{\infty} \sum_{h=0}^{\infty} \frac{\lambda^{2 h+N-2}}{N !} W_{g, N}(\beta)-\frac{W_{0,1}}{\lambda}
$$

Since neither $F(\beta)_{\text {torus }} \equiv F_{1}(\beta)$ nor $W_{0,2}(\beta)$ are weighted by $\lambda$, we should keep them in the full exponential, multiplying all terms in the weak-coupling expansion; the generic term $\mathcal{Z}_{m}^{\prime}(\beta)$ will then be of the form

$$
\mathcal{Z}_{m}(\beta)=e^{-\beta F(\beta)_{\text {torus }}-\frac{1}{2} W_{0,2}(\beta)}\left[\sum_{r, s} D_{\left(g_{i}, h_{i}, N_{i}\right)} F_{g_{1}}(\beta) \ldots F_{g_{r}}(\beta) W_{h_{1}, N_{1}}(\beta) \ldots W_{h_{s}, N_{s}}(\beta)\right]
$$

where $D_{\left(g_{i}, h_{i}, N_{i}\right)}$ are some combinatorial factors and the sum is restricted to $g_{i}>1$, $2 h_{i}+N_{i}>2$, and $\sum_{k=1}^{r}\left(2 g_{k}-2\right)+\sum_{\ell=1}^{s}\left(2 h_{\ell}+N_{\ell}-2\right)=m$. In general we find that the contributions to the energy density in the one-instanton sector mix Riemann surfaces with and without boundaries. This has to be so, since both connected and non-connected FeynmanPolyakov diagrams appear in the weak-coupling expansion of $\mathcal{Z}(\beta)=\exp [-\beta F(\beta)]$.

Using the techniques of Appendix B, we can get an estimate of the coefficients $\Omega_{m}^{\prime}(E)$ in the expansion of the density of states. The first term in the one-instanton sector is

$$
\Omega_{0}^{\prime}(E)=\int_{c-i \infty}^{c+i \infty} \frac{d \beta}{2 \pi i} e^{-\beta F(\beta)_{\text {torus }}-\frac{1}{2} W_{0,2}(\beta)} e^{\beta E} .
$$

The first singular point contributing to this integral comes from the leading singularity of $F(\beta)_{\text {torus }}$, the Hagedorn inverse temperature $\beta_{H}$. As a matter of fact, we know that $W_{0,2}(\beta)$ is regular at $\beta_{H}$ (its rightmost singularity in the complex $\beta$ plane lies at $\beta_{H} / 2$ ), so, modulo overall numerical factors, we get a term analogous to the leading contribution 
of the perturbative part of $\Omega(E)$, except for the exponetial suppression by the instanton action. Putting the perturbative and non-perturbative terms together, we end up with the following leading behaviour for $\Omega(E)$ as $E \rightarrow \infty$ (up to the one-instanton level)

$$
\Omega(E) \sim\left(C_{0}+C_{0}^{\prime} e^{-|Q| / \lambda}\right) \frac{e^{\beta_{H} E}}{E^{\frac{d}{2}+1}},
$$

where $C_{0}$ and $C_{0}^{\prime}$ are two numerical constants. The conclusion is that the only effects of D-instantons in the leading large- $E$ behaviour of $\Omega(E)$ is in changing the overall $E$ independent normalization 13 . This only amounts to a constant shift in the microcanonical entropy and thus has no consequences for the thermodynamics. More interesting for the physics is the contribution to the inverse Laplace transform coming from the first singular points of $\mathcal{Z}(\beta)$ to the left of the Hagedorn point, $\beta_{c}=\beta_{H} / 2$, which is common to both $F(\beta)_{\text {torus }}$ and $W_{0,2}(\beta)$.

Here it is convenient to split the discussion between the purely bosonic case and the IIB D-instantons that will be considered in the next subsection. Since infrared divergences in the Dirichlet sector have a vanishing effective dimension, the one-loop open string diagram leads to a logarithmic singularity, as we already saw in eq. (3.4). When defined with the natural normalization inherited from T-duality, the full exponential of the cylinder diagram entering (3.10) has a simple critical exponent; we simply find

$$
e^{-\frac{1}{2} W_{0,2}(\beta)} \sim\left(\beta-\beta_{c}\right)^{-1 / 2}
$$

For $\exp \left[-\beta F(\beta)_{\text {torus }}\right]$ we give the general expansion in eq. (B.1).

To complete the analysis we need to study the polynomial in (3.9). The critical behaviour of $F_{g}(\beta)$ is given in eq. (3.7) and for $W_{h, N}(\beta)$ we have that, above the annulus diagram, all infrared singularities in the Dirichlet open string channel are governed by singular propagators. Now, however, the effective dimensions of the infrared singularity is $d_{\text {eff }}=0$, so for $n$ soft strips $(n<N)$ we have

$$
W_{g, N}(\beta) \sim\left[\int_{0}^{\infty} d t e^{-t\left(\beta-\beta_{c}\right)}\right]^{n} \sim\left(\beta-\beta_{c}\right)^{-n}
$$

13 Actually, the fact that there is no D-instanton-induced singularities at $\beta_{H}$ implies that the structure of the correction would be the same for all the coefficients $C_{g}$ in a perturbative expansion of $\Omega(E)$. 
Because of the rational critical exponent in (3.12) we have that, contrary to the perturbative case, now it is the single-valued part in (B.1) the one contributing to the integral along the branch cuts. From (B.1), (3.12) and (3.13) we find that $(m=2 h+N-2)$ :

$$
\mathcal{Z}_{m}^{\prime}(\beta)=e^{-\beta F(\beta)_{\text {torus }}-\frac{1}{2} W_{0,2}(\beta)} W_{h, N}+\text { less divergent terms } \sim\left(\beta-\beta_{c}\right)^{-n+\frac{1}{2}},
$$

where $n<N$ is the number of soft strips; as a consequence the leading terms in the large$E$ expansion in the instanton-induced density of states associated with the singularity at $\beta_{c}=\beta_{H} / 2$ will be of order

$$
\lambda^{2 g+N-2} e^{-|Q| / \lambda} E^{n-\frac{1}{2}} e^{\beta_{H} E / 2} .
$$

A negative critical exponent with no bound! This means that, even being strongly suppressed by the string coupling constant, the contribution from surfaces with an arbitrarily large number of boundaries will be the ones dominating asymptotically the high-energy regime at the first subleading singularity, $\beta_{c}=\beta_{H} / 2$; moreover, since the critical exponent is negative they tend to make the specific heat positive. It is, however, difficult to decide a priori to what extent (3.14) is quantitatively important, because these terms are down with respect to the contributions from the Hagedorn point (3.11) by two exponential suppression factors: one in coupling $e^{-|Q| / \lambda}$ and, the most important, in energy $e^{-\beta_{H} E / 2}$.

It is perhaps more natural to interpret (3.14) as a pathology of the bosonic string D-instanton expansion. The fact that $n$ has no bound means that the large- $E$ limit does not commute with the weak-coupling expansion and therefore we must regard (3.14) as a severe "infrared catastrophe" of the dilute D-instanton gas of the bosonic microcanonical ensemble. Notice that this pathology is not a general problem of string perturbation theory, but rather a specific issue of the Dirichlet-instanton construction, since it is only the Dirichlet channel singularities that produce negative unbounded critical exponents.

We can summarize the situation by saying that whenever a given Riemann surface degeneration produces a positive specific heat critical exponent, then the weak-coupling expansion and the high-energy limit fail to commute, rendering the whole dilute instanton approach useless. In other words, the occurrence of a negative specific heat phase at high energies seems to be characteristic of the weak-coupling expansion, even when supplemented by a dilute gas of instantons. In the next paragraphs we try to argue that such pathologies are absent for the type-II case, and therefore dilute D-instantons would not induce any qualitative changes in the standard perturbative lore. 


\subsection{Type-IIB Strings}

For a number of reasons, we expect the type-II D-instanton gas to be much better behaved. First of all, the theory is (modulo rigour) finite at zero temperature. In the bosonic case, the instanton contributions to the vacuum energy $\Lambda \equiv F(\beta=\infty)$ are notoriously singular:

$$
\Lambda=\Lambda_{\text {perturbative }}-e^{-|Q| / \lambda} e^{-\Gamma_{1}^{\prime}(\beta=\infty)}+\mathcal{O}\left(e^{-2|Q| / \lambda}\right)
$$

We see that, due to the exponentiation effect of the instanton calculus, the subtracted free energy $F(\beta)-\Lambda$ is still afflicted from the zero-temperature tachyons running in handles contributing to $\Gamma_{1}^{\prime}(\beta=\infty)$. In the supersymmetric case, on the other hand, both $\Lambda_{\text {perturbative }}$ and $\Gamma_{1}^{\prime}(\infty)$ should vanish; a space-time fermionic zero mode develops in front of the full non-perturbative contribution, since the D-instanton breaks half the supersymmetries of the type-II vacuum (see Appendix A).

Another property of the type-IIB strings that makes the instanton expansion much better behaved is the absence of classical interactions between instantons. In spite of this, instantons and anti-instantons do interact with a Coulomb tail at long distances and, at the string scale, the interaction between them blows up in a Hagedorn-like fashion. As we explained above, this problem is avoided by working with explicit hard cores of radius $\ell_{0}=\beta_{H} / 2$. There is, in any case, a potential source of temperature-dependent divergences due to a winding open string joining a D-instanton with an anti-D-instanton separated by a distance $\ell$. At finite temperature, in the imaginary time formalism, one can view such a configuration as a periodic array of instantons with separation $\beta$ interacting with a similar parallel array of anti-instantons. Temperature-dependent singularities in their interaction potential appear whenever there is an open string stretching between these two arrays with length smaller than $\beta_{H} / 2$. Because we are in flat Euclidean space, the length of such a string will be simply $\ell^{2}+n^{2} \beta^{2}$, with $n$ an integer, the winding number; because we are placing the restriction $\ell>\ell_{0}=\beta_{H} / 2$, the presence of the hard core guarantees that there are no divergences for any value of the inverse temperature $\beta$.

Let us recall the detailed structure of the cancellation in the interaction between instantons. The world-sheet Hamiltonian in the Dirichlet open string channel takes the form $\Delta_{s}=\left(L_{0}-a\right)_{s}$ with $a_{s}$ the standard intercept for the corresponding spin structure: 


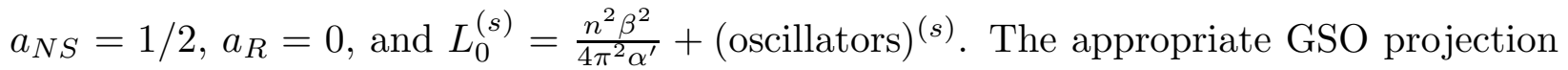
leading to a vanishing loop diagram is $\left(w=e^{-t}\right)$

$$
\begin{aligned}
W_{0,2}= & -\frac{1}{4} \int_{0}^{\infty} \frac{d t}{t}\left[\operatorname{Tr}_{N S}\left(1-(-1)^{F}\right) e^{-t \Delta_{N S}}-\operatorname{Tr}_{R}\left(1-(-1)^{F}\right) e^{-t \Delta_{R}}\right] \\
= & -\frac{1}{4} \int_{0}^{\infty} \frac{d t}{t} \sum_{n \in \mathbf{Z}} e^{-t \frac{n^{2} \beta^{2}}{4 \pi^{2} \alpha^{\prime}}}\left[w^{-1 / 2} \prod_{m=1}^{\infty}\left(\frac{1+w^{m-1 / 2}}{1-w^{m}}\right)^{8}\right. \\
& \left.-w^{-1 / 2} \prod_{m=1}^{\infty}\left(\frac{1-w^{m-1 / 2}}{1-w^{m}}\right)^{8}-16 \prod_{m=1}^{\infty}\left(\frac{1+w^{m}}{1-w^{m}}\right)^{8}\right]=0 .
\end{aligned}
$$

Upon modular transformation $s=-\log q=-2 \pi^{2} / \log w=2 \pi^{2} / t$, we obtain the appropriate GSO projection in the closed string channel, where the vanishing of (3.16) is explicitly seen as a result of the BPS balance between the purely bosonic states coupling to the (bosonic) D-instanton.

Alternatively, using a Green-Schwarz formulation with the standard $\mathbf{8}_{\mathbf{s}}$ of $S O(8)$ of space-time fermions $S^{a}(z, \bar{z})$, we have a single open string vacuum with the structure $\left|\mathbf{8}_{\mathbf{v}}\right\rangle \oplus\left|\mathbf{8}_{\mathbf{c}}\right\rangle$. The first term represents bosonic ground states and the second one fermionic ground states, i.e. $(-1)^{F}\left|\mathbf{8}_{\mathbf{v}}\right\rangle=\left|\mathbf{8}_{\mathbf{v}}\right\rangle$ and $(-1)^{F}\left|\mathbf{8}_{\mathbf{c}}\right\rangle=-\left|\mathbf{8}_{\mathbf{c}}\right\rangle$, and $F$ now represents the space-time fermion number. Then we have the representation

$$
W_{0,2}=-\frac{1}{2} \int_{0}^{\infty} \frac{d t}{t} \operatorname{Tr}(-1)^{F} e^{-t \Delta_{G S}}=-\frac{1}{2} \int_{0}^{\infty} \frac{d t}{t} \prod_{m=1}^{\infty}\left(\frac{1+w^{m}}{1+w^{m}}\right)^{8} \cdot \operatorname{Tr}_{\text {vacua }}(-1)^{F}=0 .
$$

Formally, we can envisage a Ward identity in the following way: let $\mathcal{H}_{\text {open }}^{D}$ be the physical Hilbert space of the open Dirichlet string, and split it into bosonic and fermionic component\$14 $\mathcal{H}_{B} \oplus \mathcal{H}_{F}$, related by the unitary operator $\mathcal{U}_{s s}$ carrying the representation of supersymmetry in the physical Hilbert space $\mathcal{U}_{s s} \mathcal{H}_{B}=\mathcal{H}_{F}$. Now

$$
\operatorname{STr}_{\mathcal{H}} e^{-t \Delta} \equiv \operatorname{Tr}_{\mathcal{H}_{B}} e^{-t \Delta}-\operatorname{Tr}_{\mathcal{H}_{F}} e^{-t \Delta}=\operatorname{Tr}_{\mathcal{H}_{B}}\left(e^{-t \Delta}-\mathcal{U}_{s s}^{-1} e^{-t \Delta} \mathcal{U}_{s s}\right)=0
$$

since $\left[\mathcal{U}_{s s}, \Delta\right]=0$. Heuristically, all perturbative non-renormalization theorems in string theory have this structure. If a Riemann surface with at least one closed loop (in a closed or open string channel) has an unbroken space-time supersymmetry, implemented by the

14 The notion of space-time fermion in the zero-dimensional space-time of the open string channel is provided by the continuation from higher D-branes. In particular, at the massless level, we just have the dimensional reduction of $D=9+1$ supersymmetry down to zero dimensions. 
operator $\mathcal{U}_{s s}$, then the diagram vanishes by an analogous argument. One would just open the loop inserting unity and write the full diagram in operator form as

$$
\operatorname{STr}_{\mathcal{H}_{\text {open }}^{D}}\left[\Delta^{-1} \mathcal{O}\left(\Sigma^{\prime}\right) \Delta^{-1}\right]=\operatorname{Tr}_{\mathcal{H}_{B}}\left[\Delta^{-1}\left(\mathcal{O}\left(\Sigma^{\prime}\right)-\mathcal{U}_{s s}^{-1} \mathcal{O}\left(\Sigma^{\prime}\right) \mathcal{U}_{s s}\right) \Delta^{-1}\right]
$$

where $\mathcal{O}\left(\Sigma^{\prime}\right)$ represents the operator insertion of the rest of the Riemann surface. This clearly vanishes if $\left[\mathcal{U}_{s s}, \mathcal{O}\left(\Sigma^{\prime}\right)\right]=0$, i.e. if the Riemann surface has the unbroken $\mathcal{U}_{s s^{-}}$ supersymmetry 15 . In a covariant formalism we must realize $\mathcal{U}_{s s}$ in terms of a contour integral of the corresponding supersymmetric current, and then we would prove (3.19) by a contour deformation argument. To be more precise, taking into account the spin structures, the right-hand side of (3.19) is not always zero but rather leads to a boundary term on the perturbative moduli space of Riemann surfaces, which we tacitly discard in these conceptual digressions.

The cylinder or annulus diagram vanishes in (3.16) or (3.17) at finite temperature, exactly as it does at zero temperature. In other words, this particular diagram is "supersymmetric", even if we know that supersymmetry is broken by the thermal boundary conditions. Notice that, in individual diagrams, supersymmetry is broken because boundary conditions for fermionic loops now differ from those of bosonic loops (i.e. antiperiodic rather than periodic). However, in the cylinder diagram, the only states propagating around the thermal loop are the closed string states (it is the cylinder that wraps around the thermal circle before it ends again at the instanton location). But all states in the closed string channel are bosonic, because the D-instanton boundary state (2.4) is bosonic. This is right as long as the temperature is not too small, in which case we have to consider the fermionic quasi-zero modes discussed in Appendix A, inducing fermionic boundary states. However, in the vicinity of the critical temperature, such modes have a gap of the order of the string scale, and only the bosonic D-instantons should be considered.

Since the cylinder diagram is purely bosonic in the closed string channel, the GSO projection is the same as in the zero-temperature case, and we therefore have the standard vanishing result. Clearly, as soon as we have at least one closed-string loop which could propagate fermions around the thermal circle, the diagram does break supersymmetry.

15 It is important that we have at least one closed loop in the Riemann surface, so that for example there is no vanishing theorem for the disk diagram. This is just fine, since the disk determines the D-instanton action, and it is certainly non-zero in the type-IIB theory. 
However, the D-instanton is still coupling only to bosonic states, so that if we isolate the open string strip between two boundaries we still can represent the amplitude as in (3.19)

$$
W_{g, N}=\operatorname{STr}_{\mathcal{H}_{\text {open }}^{D}}\left(\Delta^{-1} \mathcal{O}\left(\Sigma_{g, N-2}\right) \Delta^{-1}\right),
$$

where now $\mathcal{O}\left(\Sigma_{g, N-2}\right)$ represents the operator insertion of the rest of the Riemann surface, having exactly one less open-string loop, which accounts for two holes. Now, as long as we have $g>0$, some closed-string fermions will wrap the thermal circle and therefore $\left[\mathcal{U}_{s s}, \mathcal{O}\left(\Sigma_{g, N-2}\right)\right] \neq 0$, i.e. $W_{g, N}$ cannot be argued to vanish on the basis of a supersymmetric Ward identity. However, by world-sheet locality, it is still true that no fermions run in the closed channel of the selected strip. So, locally, the GSO projection in this part of the Riemann surface must be the same as at zero temperature. This means that the open string tachyon in the NS sector triggered at $\beta_{c}=\beta_{H} / 2=\pi \sqrt{2 \alpha^{\prime}}$ by $\Delta_{\text {open }}^{D}(N S)=\frac{\beta^{2}}{4 \pi^{2} \alpha^{\prime}}-\frac{1}{2}=0$, is again projected out by the same GSO projection applied in (3.16). Working in the Green-Schwarz formalism, the propagators entering (3.20) would have the structure $\Delta_{G S}=\frac{n^{2} \beta^{2}}{4 \pi^{2} \alpha^{\prime}}-a+$ osc, with integrally moded fermions $S^{a}$ and then $a=0$.

So the final conclusion is that in the type-IIB superstring all temperature-dependent divergences in the one-instanton sector are associated exclusively with the degeneration of closed string handles. This means that there are no new string states becoming massless at the Hagedorn temperature besides the tachyonic winding mode already present in the zero-instanton sector. The obvious consequence is that we get the same critical exponents for $\mathcal{Z}(\beta)$ as in the zero-instanton sector; the only change in the large- $E$ expansion of $\Omega(E)$ then is a modification of the coefficients of the asymptotic series, much in the fashion of eq. (3.11). Because of the absence of singularities in the partition function associated with D-instantons, there are no terms in $\Omega(E)$ contributing to positive values of the specific heat. This makes the weak-coupling expansion well defined for the type-IIB strings, but on the other hand seems to indicate that the introduction of D-instanton effects does not imply any qualitative modification in the thermodynamics of type-IIB superstrings near the Hagedorn temperature, as it is given by string perturbation theory in the microcanonical ensemble. 


\section{Mean Field Analysis of the Hagedorn Transition}

Having studied the effects of D-instantons in the microcanonical description of string thermodynamics, we now turn to an analysis of the instanton effects on the Hagedorn transition. In the previous section we saw that, at least for the case of the type-IIB superstring, single D-instanton effects do not introduce new divergences; therefore, we can assume the winding closed string tachyon to be the only relevant order parameter of the Hagedorn phase transition. In what follows, we will include dilute instanton effects in the mean field approximation of [30], which leads to a first-order transition in perturbation theory.

\subsection{Mean Field Approximation}

Following Atick and Witten [30], we consider the effective long-wavelength dynamics of the complex tachyon field denoted by $\sigma$, which is assumed to nucleate the phase transition. For this purpose we use a mean field approach, considering $\sigma$ as a smooth background field in the spatial dimensions, and we integrate out fluctuations for all the approximately massless degrees of freedom. At large spatial scales, only non-derivative interactions are important and we have the following effective nine-dimensional Lagrangian as a starting point

$$
\begin{aligned}
\mathcal{L}_{9}= & -\sigma^{*} \vec{\partial}^{2} \sigma+m_{\mathrm{eff}}(T)^{2} \sigma \sigma^{*}+\lambda^{2} T \alpha^{\prime 3} c\left(\sigma \sigma^{*}\right)^{2} \\
& -\frac{1}{2} \sum_{f} \varphi_{f}\left(-\vec{\partial}^{2}+m_{f}^{2}\right) \varphi_{f}+\lambda \alpha^{\prime} \sqrt{T} \sum_{f} c_{f} \sigma^{*} \sigma \varphi_{f}+\ldots
\end{aligned}
$$

where we have included the quartic self-coupling of the tachyon induced at tree-level, $c \sim\left\langle V_{\sigma} V_{\sigma} V_{\sigma^{*}} V_{\sigma^{*}}\right\rangle$ is of order unity and positive, since we do not expect instabilities at zero temperature. The ellipsis stands for higher-dimension couplings and higher-derivative terms. The effective mass squared $m_{\text {eff }}^{2}$ vanishes linearly at the critical temperature 6 , and the sum in (4.1) runs over the set of almost massless fields at the singularity (radiatively or instanton-induced masses). Integrating out the fields $\varphi_{f}$ at tree-level we obtain an effective potentialit

$$
V_{\mathrm{eff}}\left(\sigma, \sigma^{*}\right)=m_{\mathrm{eff}}(T)^{2} \sigma^{*} \sigma+\lambda^{2} T \alpha^{\prime 3} c\left(\sigma \sigma^{*}\right)^{2}-\frac{1}{2} \lambda^{2} T \alpha^{\prime 2} \sum_{f} c_{f}^{2}\left\langle\sigma^{*} \sigma\left|\left(-\vec{\partial}^{2}+m_{f}^{2}\right)^{-1}\right| \sigma^{*} \sigma\right\rangle
$$

16 In the type-IIB theory we have $m_{\text {eff }}^{2}=\frac{\beta^{2}}{4 \pi^{2} \alpha^{\prime 2}}-\frac{4}{\alpha^{\prime}}$ at tree-level.

17 We can tune the string coupling $\lambda$ to be artificially small. In fact, we must do so in order to avoid the Jeans instability, which would invalidate any thermodynamical approach [30]. 
and in the infrared limit we are left with the contact term

$$
V_{\mathrm{eff}}\left(\sigma, \sigma^{*}\right) \rightarrow m_{\mathrm{eff}}(T)^{2} \sigma^{*} \sigma+\lambda^{2} T \alpha^{\prime 3} c\left(\sigma \sigma^{*}\right)^{2}-\frac{1}{2} \lambda^{2} T \alpha^{\prime 2} \sum_{f} \frac{c_{f}^{2}}{m_{f}^{2}}\left(\sigma^{*} \sigma\right)^{2} .
$$

The character of the phase transition is therefore determined by the relative values of the ratios of tree-level couplings to masses $c_{f}^{2} / m_{f}^{2}$. Fields $\varphi_{f}$ with a real coupling $c_{f}$ to the NS-NS tachyon $\sigma$ contribute negative quartic terms to (4.2), and if dominating it follows that the phase transition is first order, since an instability to the condensation of the field $\sigma$ sets in when we still have $m_{\text {eff }}^{2}(T)>0$. Higher-order terms are expected to stabilize $V_{\text {eff }}$ at large field strength. If one of the fields $\varphi_{f}$ is exactly massless, then $m_{f}^{2}$ should be replaced by some infrared cutoff, say the inverse volume of the spatial box. This is perfectly reasonable since we are simply exhibiting the qualitative character of the transition as first order as opposed for example to a second-order transition, where the order parameter only condenses when it becomes tachyonic.

On the other hand, if $c_{f}$ is imaginary then $\varphi_{f}$ is a sort of "scalar gauge field" with respect to which $\sigma$ has charge $\left|c_{f}\right|$. Such terms induce a positive quartic term in (4.2) and, if dominating, we have a second-order transition instead. Notice that, since we are assuming $c>0$, the bare potential tends to produce a second-order scenario.

The analysis of [30] dealt only with the dilaton. In fact, it is easy to argue that the other universal NS-NS massless field, the antisymmetric tensor $B_{\mu \nu}$, is irrelevant for the infrared limit in (4.2). The fields $\sigma$ (resp. $\sigma^{*}$ ) have winding number +1 (resp. -1 ) so that they have \pm charge with respect to the nine-dimensional gauge field $A_{i}=B_{i 0}$. However, the corresponding electromagnetic current $\sigma^{*} \vec{\partial} \sigma$ contains a derivative, which makes it irrelevant in the infrared.

These considerations lead us to focus just on the axion-dilaton scalar sector of the type-IIB theory. In perturbation theory the dilaton couples at tree-level to the tachyon field, $c_{\phi}$ is of order unity, and a radiative mass is generated at one-loop order $m_{\phi}^{2} \sim \lambda^{2} / \alpha^{\prime}$, for temperatures close to the critical Hagedorn point 18 . Then the dilaton contribution to quartic terms in (4.2) is

$$
\delta V_{\text {dilaton }} \sim-\left(\alpha^{\prime}\right)^{5 / 2}\left(\sigma \sigma^{*}\right)^{2}
$$

18 For $T \leq \alpha^{\prime-1 / 2}$ we can easily estimate the self-energy correction in the low-energy effective theory (4.1) keeping also the dilaton Kaluza-Klein modes of mass $\sim T$. A simple dimensional analysis of the loop diagram gives $m_{\phi}^{2} \sim \lambda^{2} T^{6} \alpha^{\prime 2}$. More detailed treatments of mass corrections in the full-fledged string theory can be found for example in [37]. 
for $T \sim \alpha^{\prime-1 / 2}$, and we see that this term, at weak coupling, clearly dominates the bare tachyon potential and settles the scenario of a first-order transition for the case of the bosonic or heterotic string. Since both $c_{\phi}$ and $m_{\phi}^{2}$ are sizeable in perturbation theory, instanton corrections make a negligible contribution to (4.3).

In the type-IIB theory this is not the end of the story because of the axion field. Here both the coupling to the tachyon $c_{a}$ and the induced mass $m_{a}$ vanish in perturbation theory, as a result of the classical $S L(2, \mathbf{R})$ symmetry of the ten-dimensional type-IIB theory, which in particular involves $a \rightarrow a+$ constant, and forbids non-derivative couplings of the axion in perturbation theory. This symmetry is broken by D-instantons and in general by the existence of D-branes to a discrete $S L(2, \mathbf{Z})$ symmetry, so that periodic couplings of the axion can be generated at the non-perturbative level. Since the derivative of the axion $\partial_{\mu} a$ behaves like a $U(1)$ field strength -in particular it is dual to the 9 -form field strength $F_{9}$ coupling to 7 -branes- it is at least possible that a non-perturbatively generated tachyon-axion coupling would have $c_{a}$ pure imaginary.

We see that the sign and magnitude of the ratio $c_{a}^{2} / m_{a}^{2}$ is completely determined by non-perturbative physics, and we conclude that the D-instanton analysis is crucial to decide the order of the transition in the type-IIB theory.

\subsection{Axion Couplings}

The classical symmetry $a \rightarrow a+$ constant, is embodied in type-IIB string perturbation theory in the fact that $\mathrm{R}-\mathrm{R}$ vertex operators are "longitudinal" or, more precisely, correspond really to field strengths rather than to the "photon" fields, and correlation functions vanish at zero momentum 19 . Even more generally, this follows from separate left-right conservation of the world-sheet fermion number.

19 For example, the axion vertex operators in the $\left(q_{1}, q_{2}\right)$ picture read

$$
V_{a}^{\left(q_{1}, q_{2}\right)}=p_{\mu}\left(\gamma^{\mu}\right)_{A B} V_{q_{1}}^{A}(z) V_{q_{2}}^{B}(\bar{z})
$$

and, in the canonical $q=-1 / 2$ picture, $V_{-1 / 2}^{A}(z)=e^{-\phi(z) / 2} S^{A}(z) e^{i p X(z)}$, where $\phi(z)$ here denotes the bosonized ghost field and has no relation with the dilaton. Then, a simple OPE check shows that mass corrections $\left\langle V_{R R} V_{R R}\right\rangle$ and vertex amplitudes $\left\langle V_{R R} V_{\sigma} V_{\sigma^{*}}\right\rangle$ induce derivative interactions after we integrate the vertex operators over the world-sheet, so that all amplitudes vanish at zero momentum. 
Such arguments could in principle fail when considering D-instantons, since the integral over instanton locations might pick up surface terms coming from the derivative couplings, leading to a constant in the zero-momentum limit. Now, there is no a priori reason for amplitudes like

$$
J_{\text {coll }} A e^{-|Q| / \lambda} \lambda^{2} \int d x_{0}\left\langle V_{\sigma} V_{\sigma^{*}} V_{a} \mid I, x_{0}\right\rangle_{\text {disk }}
$$

contributing to cubic $\sigma \sigma^{*} a$ couplings to vanish in the zero-momentum limit. In fact, the leading instanton contribution to the cubic axion-tachyon coupling has no extra powers of the string coupling constant and it is given by

$$
J_{\text {coll }} A e^{-|Q| / \lambda} \int d x_{0}\left\langle V_{\sigma} \mid I, x_{0}\right\rangle_{\text {disk }}\left\langle V_{\sigma^{*}} \mid I, x_{0}\right\rangle_{\text {disk }}\left\langle V_{a} \mid I, x_{0}\right\rangle_{\text {disk }},
$$

which clearly vanishes: the net winding number introduced in the disk by the tachyon vertex operators cannot flow through the instanton, since we have Dirichlet boundary conditions on the boundary of the disk for all directions.

The next correction scales with an overall power of $\lambda$ and has the form

$$
J_{\text {coll }} A e^{-|Q| / \lambda} \lambda \int d x_{0}\left\langle V_{\sigma} V_{\sigma^{*}} \mid I, x_{0}\right\rangle_{\text {disk }}\left\langle V_{a} \mid I, x_{0}\right\rangle_{\text {disk }}
$$

In this case, it is clear that we have a non-trivial result because the $\sigma-\sigma^{*}$ pair couples dominantly to the $\mathrm{D}$-instanton via dilaton exchange, and the axion couples directly to the D-instanton.

On general grounds, the disk "tadpole" or one-point function of a vertex operator in the background of the instanton is related, after propagator amputation, to the classical profile of the associated field in the instanton configuration. For example, for a scalar field $\Phi(x)$ we can write, in a somewhat symbolic fashion,

$$
\left(-\partial_{x}^{2}+m_{\Phi}^{2}\right) \Phi\left(x-x_{0}\right)_{c \ell} \sim \int d p e^{i p x}\left\langle V_{\Phi}(p) \mid I, x_{0}\right\rangle_{\text {disk }}
$$

This is interesting because the classical profile of the axion field $a_{c \ell}$, which is purely imaginary, enters (4.6) directly, so that we have a specific source for imaginary contributions to the axion coupling $c_{a}$.

A detailed analysis of the size and sign of the instanton-induced axion-tachyon couplings is complicated. Fortunately for us, the structure of (4.2) implies that, as long as an axion mass is generated in the one-instanton sector, the dilaton dominance of the 
phase-transition dynamics is ensured, and therefore the standard first-order picture would not be modified. This follows from the fact that, up to powers of the string coupling, $\left|c_{a}\right|^{2} \leq e^{-2|Q| / \lambda}$, because $c_{a}$ is at most generated in the one-instanton sector. On the other hand, if the mass squared is generated at the one-instanton level we have $m_{a}^{2} \sim e^{-|Q| / \lambda}$ and thus the relevant ratio

$$
\frac{\left|c_{a}\right|^{2}}{m_{a}^{2}} \leq e^{-|Q| / \lambda}
$$

This implies that the dilaton dominates independently of the sign of $c_{a}^{2}$. The axion would be competitive only if its mass were generated at the two-instanton level. In such a case the size of the ratio $\left|c_{a}\right|^{2} / m_{a}^{2}$ would depend on the power dependence in the string coupling.

Now, from the general discussion of section 2.3 we know that long-range Coulomb interactions of instantons without fermion zero modes generate periodic potentials for the axion. The discussion in Section 2.3 is obscured by the fact that D-instanton gases do not really form a plasma, because dilaton exchange exactly cancels the instanton-instanton repulsion. The result was a runaway potential for the dilaton that is only stabilized at vanishing coupling. However, at finite temperature this picture is only valid in the intermediate scales between the string scale and the induced mass of the dilaton $m_{\mathrm{dil}}^{2} \sim \lambda^{2} T^{6} \alpha^{\prime 2} \sim \lambda^{2} / \alpha^{\prime}$, which can be considerably lower than the string scale for weak coupling, even at the critical temperature. Below this energy scale the dilaton exchange is screened and we have a real Coulomb plasma. The runaway of the dilaton is in turn stabilized by the radiatively induced potential and we end up with an axion mass at the one-instanton order

$$
m_{a}^{2}=2 A J_{\text {coll }} Q^{2} e^{-|Q| / \lambda}+\ldots
$$

so we conclude that the standard first-order scenario is not modified.

\section{Concluding remarks}

In the present paper we have tried to clarify the relevance of D-instanton effects in string thermodynamics, with special interest on their influence in the critical behaviour of the string collective. We have seen that D-instantons can induce strong infrared singularities in thermodynamical quantities. Indeed, for purely bosonic D-instantons, the whole instanton expansion seems ill-defined in the microcanonical approach. On the other hand, type-IIB D-instantons are smooth within the dilute approximation, although they do not qualitatively modify the perturbative picture near the Hagedorn point. 
Still, the physics of dense "liquids" containing instantons and anti-instantons remains obscure, and the existence of thermal singularities in the type-IIB string analogous to those found in the bosonic case cannot be completely excluded. Anyhow, the corresponding critical points are necessarily beyond the Hagedorn temperature, and it is quite unlikely that they are physically relevant at a quantitative level. This would be very odd on physical grounds, because we expect instanton physics to produce very small effects at weak coupling, when competing with perturbative contributions. The opposite situation would rather be interpreted as a breakdown of the semiclassical expansion.

A partial exception is the contribution of the type-IIB axion to the nucleation of the Hagedorn transition, since both its mass and non-derivative couplings are generated by finite-temperature instantons. We have found in the mean field approximation that the dilaton is still dominating and the first-order scenario of [30] is not modified at this level.

It is somewhat disappointing that D-instantons do not seem to solve the "Hagedorn problem". However, in retrospect this is a rather natural conclusion in view of the above comments. It means that the change of degrees of freedom taking place is more radical than a simple addition of non-perturbative semiclassical states.

Finite temperature remains an interesting arena for the study of D-instantons and D-branes. Specially interesting are the infrared effects that are otherwise forbidden by supersymmetry, such as the scalar potentials generated by long-range interactions. At finite temperature, supersymmetry is only broken "softly", because the gravitinos remain

in the spectrum with masses of order $\beta^{-2}$. We have seen that this is important for the consistency of the D-instanton expansion.

\section{Acknowledgements}

It is a pleasure to thank E. Álvarez, L. Álvarez-Gaumé, D.J. Gross, R. Emparan, I.R. Klebanov, M. Laucelli, T. Ortín, M.A.R. Osorio, J. Puente-Peñalba and F. Quevedo for useful discussions and suggestions. The work of M.A.V.-M. was partially supported by the Spanish Science Ministry and by a Basque Government Postdoctoral Fellowship. 


\section{Appendix A. Fermionic Quasi-zero Modes}

Fermionic collective coordinates are easily implemented in the light-cone formalism where explicit expressions for the supersymmetry charges are available [24] [27] [38]. The full super-boundary state is given by

$$
|I, x, \theta\rangle=e^{i \theta Q_{\text {broken }}}|I, x\rangle,
$$

where $|I, x\rangle$ is the bosonic boundary state (2.4), and the fermionic superpartners are generated by the broken supersymmetry in the standard fashion. For a given type-IIB instanton there are 16 independent fermionic collective coordinates and, expanding (A.1), we obtain the full multiplet of BPS-saturated instanton boundary states, entirely analogous to an instanton superfield in the treatment of [39]. The unbroken supersymmetry fixes the measure to be

$$
d \mu=d^{10} x d^{16} \theta J_{\text {coll }} .
$$

If $\theta$ represent quasi-zero modes, we induce the corresponding fermion exchange interactions upon $\theta$-integration. For example, in the $\mathrm{I}-\mathrm{A}$ sector, the leading (cylinder) static interaction in super-space is given by $\Gamma\left(x^{+}-x^{-} ; \theta^{+}, \theta^{-}\right)=\left\langle x^{-}, \theta^{-}\left|\left(\Delta_{\text {closed }}\right)^{-1}\right| x^{+}, \theta^{+}\right\rangle$. Using (A.1) and the supersymmetry algebra $\left\{Q_{+}, Q_{-}\right\}=-i \sqrt{2} \gamma \cdot \partial$ we find

$$
\Gamma\left(x^{+}-x^{-} ; \theta^{+}, \theta^{-}\right)=e^{i \sqrt{2} \theta^{-} \gamma^{\mu} \theta^{+} \partial_{\mu}} \Gamma_{I-A}\left(x^{+}-x^{-}\right),
$$

where the classical I-A overlap is given by (see for example [18])

$$
\Gamma_{I-A}(x-y)=-(2 \pi)^{4} \int_{0}^{\infty} \frac{d s}{s^{5}} e^{\frac{(x-y)^{2}}{2 \alpha^{\prime} s}} \prod_{m=1}^{\infty}\left(\frac{1+q^{2 m}}{1-q^{2 m}}\right)^{8}
$$

in the closed string channel, (here $q=e^{-s}$ ). Saturation of fermionic coordinates can be done in several ways, depending on the number of disconnected world-sheets used. For example, with 16 cylinders connecting the instanton and anti-instanton we have a product of 16 fermionic propagators with the states $Q_{-}^{\alpha}\left|I_{+}, x^{+}\right\rangle, \alpha=1, \ldots, 8, \dot{1}, \ldots, \dot{8}$ running through each cylinder. With just one cylinder we have the top (bosonic) state $Q_{-}^{1} \cdots Q_{-}^{8} Q_{-}^{\dot{1}} \cdots Q_{-}^{\dot{8}}\left|I_{+}, x^{+}\right\rangle$running.

At finite temperature, antiperiodic boundary conditions on space-time fermions lift all the supersymmetric zero modes, and therefore we should not consider fermionic collective coordinates in the generic situation. However, as $\beta \rightarrow \infty$ in the low-temperature limit we 
should recover the zero-temperature results and in particular the restoration of normalizable zero modes ensuring for example vanishing vacuum energy. The corresponding zero modes are difficult to see by a direct study of the individual diagrams $W_{1, N}$ in (2.25). The reason is that the Dirichlet construction only propagates free strings in flat space, and the zero-mode singularity in the effective action would appear only after a resummation of boundaries has been performed, in the sense of eq. (2.27). In any case, we can derive the leading suppression factor by defining collective coordinates for the quasi-zero modes at low temperature, in the sense of a constrained instanton expansion.

The idea is simple: the family (A.1) does not define classical solutions at finite temperature, but it certainly defines approximate solutions for large $\beta$. Therefore, we can still integrate over the whole fermionic family (A.1), provided we include the classical interaction with the thermal boundary conditions. As in the I-A sector, such interactions are given by propagators of the states in the BPS multiplet. In this case, a D-instanton at finite temperature is equivalent to an array of D-instantons at distance $\beta$, and we must compute the classical interaction between them induced by fermion exchange.

Denote the corresponding BPS boundary state by $\left(Q_{-}\right)^{\{p\}}\left|I_{+}\right\rangle$, where $\{p\}$ denotes a subset of $p$ indices in the $\mathbf{8}_{\mathbf{s}} \oplus \mathbf{8}_{\mathbf{c}}$ of $S O(8)$. Then, by the properties of the charges and the explicit form of (2.4) we have $\left(Q_{-}\left|I_{+}\right\rangle\right)^{\dagger}=\left\langle I_{-}\right| Q_{+}$. Therefore, a propagator wrapped around the thermal loop has the form

$$
\left(\left(Q_{-}\right)^{\{p\}}\left|I_{+}\right\rangle\right)^{\dagger} \Delta_{\text {closed }}^{-1}\left(Q_{-}\right)^{\{p\}}\left|I_{+}\right\rangle_{p}=\left\langle I_{-}\left|\left(Q_{+}\right)^{\{p\}} \Delta_{\text {closed }}^{-1}\left(Q_{-}\right)^{\{p\}}\right| I_{+}\right\rangle_{p}
$$

and indeed looks like an I-A interaction. The subindex means that fermions should be antiperiodic in the case that $p$ is odd. Using the supersymmetry algebra this is equal to

$$
2^{p / 2} \operatorname{det}_{\{p\}}(\gamma \cdot \partial) \Gamma_{p}(\beta)
$$

The determinant is over the subset $\{p\}$ of Dirac indices, and $\Gamma_{p}(\beta)$ is the $\mathrm{I}-\mathrm{A}$ overlap in the infinite array of instantons. For $p$ even, it is just the periodic sum of the elementary I-A overlaps in (A.4). For $p$ odd we have antiperiodic boundary conditions, which is easily accomplished by introducing a phase for odd wrappings of the form

$$
\Gamma_{p=\text { odd }}(\beta)=\sum_{n=0}^{\infty}(-1)^{n} \Gamma_{I-A}(n \beta) .
$$


The function $\Gamma_{I-A}$ was written above (A.4), and the antiperiodicity phase produces half integer modding after Poisson resummation in $n$.

Now, the determinant factor gives a term $\left(\partial_{\beta}\right)^{p} \Gamma_{p}$ for each cylinder. With $n_{c}$ cylinders

such that $\sum_{i=1}^{n_{c}} p_{i}=16$, and given the low-temperature scaling of $\Gamma_{p}$ we find that oneinstanton effects are suppressed in the low-temperature limit as

$$
\prod_{i}\left(\frac{\partial}{\partial \beta}\right)^{p_{i}} \Gamma_{p_{i}}(\beta) \sim \prod_{i} \beta^{-8-p_{i}}=\beta^{-8\left(n_{c}+2\right)} \leq \beta^{-24}
$$

in agreement with our expectations.

\section{Appendix B. Asymptotic form of $\Omega(E)$}

In this Appendix we will outline the calculation leading to the asymptotic expansion of $\Omega(E)$ for large values of $E$ at one loop. Following ref. [34] we compute the inverse Laplace transform (3.5) by moving $c$ to the left and deforming the contour in order to avoid the singular points of the integrand20. This demands the analytic continuation of $\mathcal{Z}_{0}(\beta)=$ $\exp \left[-\beta F(\beta)_{\text {torus }}\right]$ to the whole complex $\beta$-plane. This is a subtle step since it is known 40. that the modular-invariant representation of $F(\beta)_{\text {torus }}$ is afflicted by singularities at the self-dual length under $\beta$-duality, $\beta_{\mathrm{s}-\mathrm{d}}$, of the form $\left|\beta-\beta_{\mathrm{s}-\mathrm{d}}\right|^{a}$ with $a$ odd. Obviously, this kind of singular behaviour does not allow an analytic continuation to complex values of $\beta$. To avoid these problems we will work with the so-called S-representation of the torus free energy, which is obtained by summing up the individual contributions to the free energy from the fields contained in the string spectrum [35] and 36]. The expression so obtained can be analytically continued to complex values of the inverse temperature and, as a bonus, there are no singular points except those located on the real and imaginary axes. Actually, for real $\beta$ we have branch-point singularities located at $\beta_{n}=\beta_{H} / n(n \in \mathbf{Z})$. Taking all branch cuts to the left we decompose (3.5) into an integral along $\operatorname{Re} \beta=c$ plus the integral along the contour $C$ surrounding the branch cuts. The integral over the vertical contour will be of order $\exp (c E)$, so we can write

$$
\Omega_{0}(E)=\int_{C} \frac{d \beta}{2 \pi i} \mathcal{Z}_{0}(\beta) e^{\beta E}+\mathcal{O}\left(e^{c E}\right) .
$$

20 See [10] for a different treatment of the large- $E$ limit. 
Let us assume first that $\beta_{H} / 2<c<\beta_{H}$. In this case we can expand $\mathcal{Z}(\beta)_{\text {torus }}$ in a series of $\left(\beta-\beta_{H}\right)$ with a convergence radius equal to the distance to the closest singularity (i.e. $\left.\beta_{H} / 2\right)$. One can easily convince oneself that

$$
\mathcal{Z}_{0}(\beta) \equiv e^{-\beta F(\beta)_{\text {torus }}}=\sum_{n=0}^{\infty} a_{n}^{(1)}\left(\beta-\beta_{H}\right)^{n+\frac{d}{2}}+\sum_{n=0}^{\infty} b_{n}^{(1)}\left(\beta-\beta_{H}\right)^{n} .
$$

The contour of integration $C$ is decomposed into $C_{ \pm}$, parametrized as $\beta=\beta_{H}+e^{ \pm i \pi}$. From (B.1) it is obvious that only the multivalued part contributes to the integral, since the second term cancels out. After some straightforward manipulations we get the following asymptotic expansion

$$
\Omega_{0}(E) \sim \sin \left(\frac{\pi d}{2}\right) e^{\beta_{H} E} \sum_{n=0}^{\infty}(-1)^{n+1} a_{n}^{(1)} E^{-n-\frac{d}{2}-1} \int_{0}^{\eta E} \frac{d y}{\pi} y^{n+a} e^{-y}+\mathcal{O}\left(e^{c E}\right)
$$

with $\eta=\beta_{H}-c$. Since $d=D-1$ is odd the prefactor is non-vanishing.

Up to here we have assumed that $c$ lies between the first and the second branch point. However we can push $c$ further to the left and place it between $\beta_{M}$ and $\beta_{M+1}$. In this case we can split the contours $C_{ \pm}$into the intervals $\left(\beta_{k+1}, \beta_{k}\right)(k=1, \ldots, M)$ and compute the integral over each piece by expanding $21 \mathcal{Z}_{0}$ around the corresponding $\beta_{k}$. Each expansion has exactly the same structure as (B.1), but now with different coefficients $a_{n}^{(i)}, b_{n}^{(i)}$; again only the multivalued parts contribute. Repeating the same steps as above on each piece we finish with

$$
\Omega_{0}(E) \sim \sin \left(\frac{\pi d}{2}\right) \sum_{k=1}^{M} e^{\beta_{k} E} \sum_{n=0}^{\infty}(-1)^{n+1} a_{n}^{(k)} E^{-n-\frac{d}{2}-1} \int_{0}^{\eta_{k} E} \frac{d y}{\pi} y^{n+a} e^{-y}+\mathcal{O}\left(e^{c E}\right),
$$

where $\eta_{k}=\beta_{k}-\beta_{k+1}(k=1, \ldots, M-1)$ and $\eta_{M}=\beta_{M}-c$.

If we are only interested in the leading behaviour around each singular point $\beta_{k}$, we conclude from the preceding analysis that to a leading critical behaviour $\mathcal{Z}(\beta) \sim\left(\beta-\beta_{k}\right)^{\alpha}$ will correspond a leading asymptotic term in the density of states:

$$
\Omega(E) \sim \frac{e^{\beta_{k} E}}{E^{\alpha+1}}+\text { subleading terms. }
$$

A term like this will contribute to the specific heat with

$$
\frac{1}{c_{V}} \sim-\frac{\alpha+1}{E}\left(\beta_{k}-\frac{\alpha+1}{E}\right)^{2},
$$

which will be negative unless $\alpha<-1$.

21 This can always be done since the radius of convergence of the series expansion around $\beta_{k}$ will be equal to the distance to the closest singularity, which in our case is $\beta_{k+1}$ for every $k=1,2, \ldots$ 


\section{Appendix C. Higher-order Diagrams in Open String Theory}

Closed string theory is perturbatively defined in terms of a two-dimensional quantum field theory, defined on a closed, orientable Riemann surface. Although the actual computation of string processes beyond one loop is extremely complicated, the mathematical simplicity of some general properties of closed Riemann surfaces makes it possible to extract some information about the behaviour of the contributions from Riemann surfaces of higher genus; for example one can write explicitly the sum over classical embeddings in any correlation function or the behaviour of the string measure in some corners of the moduli space at arbitrary genus.

All this simplicity seems to be lost in open string theory, where also bordered Riemann surfaces contribute; the moduli space becomes enlarged since we can now deform both handles and boundaries. There is, however, a way in which one can relate open string diagrams to much simpler closed string ones and thus make use of standard procedures to get information about higher orders in open string perturbation theory. In this Appendix we will review the technique of doubled bordered Riemann surfaces [41], [42], [43], and apply it to prove that there are no corrections to the one-loop critical temperature in open bosonic string perturbation theory as computed from the temperature-dependent divergences associated with the boundary of the moduli space.

Given a genus- $h$ Riemann surface $\Sigma$ with $N$ boundaries, there is a canonical way of getting a compact Riemann surface $\bar{\Sigma}$ of genus $2 h+p-1$, by reflection of the original surface with respect to its boundaries. Then $\Sigma$ can be obtained back from its double $\bar{\Sigma}$ by dividing it by an antiholomorphic involution $I_{*}$ acting on the canonical homology basis of $\bar{\Sigma}$ as $I_{*} A_{i}=\Gamma_{i j} A_{j}$ and $I_{*} B_{i}=-\Gamma_{j i} B_{j}$, where $\Gamma_{i j} \in \mathbf{Z}$ and $\Gamma^{2}=1$. The expression of $\Gamma_{i j}$ can be simplified by the following choice of the canonical homology basis: let $a_{\alpha}$, $b_{\alpha}(\alpha=1, \ldots, h)$ be the homology cycles of the handles already on $\Sigma$ and $\tilde{a}_{\alpha}, \tilde{b}_{\alpha}$ their reflection with respect to the boundaries. In addition we also have $c_{l}, d_{l}(l=1, \ldots, N-1)$, the cycles of $\bar{\Sigma}$ associated with the handles we are cutting to get $\Sigma$ back. In this basis the involution acts as $I_{*} c_{l}=c_{l}, I_{*} d_{l}=-d_{l}, I_{*} a_{\alpha}=\tilde{a}_{\alpha}$ and $I_{*} a_{\alpha}=\tilde{a}_{\alpha}$. Therefore $\Gamma$ has the following antidiagonal form

$$
\Gamma=\left(\begin{array}{lll}
0 & 0 & \mathbf{1} \\
0 & \mathbf{1} & 0 \\
\mathbf{1} & 0 & 0
\end{array}\right)
$$


The antiholomorphic action of $I_{*}$ on the doubled surface $\bar{\Sigma}$ means that its period matrix $\tau$ has to satisfy $\tau=-\Gamma^{\mathrm{t}} \bar{\tau} \Gamma$; with our choice for $\Gamma$ this means

$$
\tau=\left(\begin{array}{ccc}
a & b & c \\
b^{\mathrm{t}} & i t & -\bar{b}^{\mathrm{t}} \\
-\bar{c} & -\bar{b} & -\bar{a}
\end{array}\right)
$$

where $a^{\mathrm{t}}=a, t$ is real and $c$ is anti-hermitian $\left(c=-\bar{c}^{\mathrm{t}}\right)$.

If we want to compute scattering amplitudes for open strings from the doubled surface, we need the open string measure in terms of the string measure on $\bar{\Sigma}$. The partition function for the closed bosonic string at genus $2 h+N-2$ can be written as [44]

$$
\Lambda_{2 h+N-2}=\int_{\mathcal{M}(\bar{\Sigma})} d(\mathrm{WP})_{\bar{\Sigma}}\left(\operatorname{det}\left(P^{+} P\right)_{\bar{\Sigma}}\right)^{1 / 2}\left[\frac{8 \pi^{2}}{\int_{\bar{\Sigma}} d^{2} \xi \sqrt{g}} \operatorname{det}^{\prime} \Delta_{\bar{\Sigma}}\right]^{-13} \sum_{\text {solitons }} e^{-S_{c \ell}^{\text {closed }}}
$$

where $d(\mathrm{WP})$ is the Weil-Peterson measure defined in terms of the quadratic and Beltrami differentials, and the sum over classic configurations can be written as

$$
\sum_{\text {solitons }} e^{-S_{c \ell}^{\text {closed }}(\vec{N}, \vec{M})}=\sum_{\vec{N}, \vec{M}} e^{-\frac{\beta^{2}}{4 \pi \alpha^{\prime}}(\vec{\tau} \vec{N}-\vec{M})^{\mathrm{t}}(\operatorname{Im} \tau)^{-1}(\tau \vec{N}-\vec{M})}
$$

On the other hand the open string path integral over $\Sigma$ is formally

$$
\Lambda_{h, N}^{\text {open }}=\int_{\mathcal{M}(\Sigma)} d(\mathrm{WP})_{\Sigma}\left(\operatorname{det}\left(P^{+} P\right)_{\Sigma}\right)^{1 / 2}\left[\frac{8 \pi^{2}}{\int_{\Sigma} d^{2} \xi \sqrt{g}} \operatorname{det}^{\prime} \Delta_{\Sigma}\right]^{-13} \sum_{\text {solitons }} e^{-S_{c \ell}^{\text {open }}}
$$

The mapping between the determinants in (C.3) and (C.2) has been computed in 42 with the result

$$
\begin{aligned}
\operatorname{det}\left(P^{+} P\right)_{\Sigma} & =\left(\operatorname{det}\left(P^{+} P\right)_{\bar{\Sigma}}\right)^{1 / 2} \\
\frac{\operatorname{det}^{\prime} \Delta_{\Sigma}}{\int_{\Sigma} d^{2} \xi \sqrt{g}} & =\left(\frac{\operatorname{det}^{\prime} \Delta_{\bar{\Sigma}}}{\int_{\bar{\Sigma}} d^{2} \xi \sqrt{g}}\right)^{1 / 2} R_{\bar{\Sigma}, I}^{\mp 1 / 2},
\end{aligned}
$$

where $\mp$ is for Neumann/Dirichlet boundary conditions and $R_{\Sigma, I}$ is defined by

$$
R_{\bar{\Sigma}, I}=\operatorname{det}\left[(1+\Gamma) \operatorname{Im} \tau+(1-\Gamma)(\operatorname{Im} \tau)^{-1}\right]
$$

The integration region $\mathcal{M}(\Sigma)$ is a real submanifold of the moduli space $\mathcal{M}(\bar{\Sigma})$ and therefore the square roots are holomorphic square roots. 
Special care is needed in the case of the Weil-Peterson measure. Using the complex structure $J$ on $\bar{\Sigma}$, which anticommutes with $I_{*}$, one can write a base of quadratic differentials [42] $\left\{S_{i}, J S_{i}\right\}$, where $I_{*} S_{i}=S_{i}$. In the same way this can be done also for a base of Beltrami differentials. Following the details in [42] one finds that

$$
d(\mathrm{WP})_{\bar{\Sigma}}=d(\mathrm{WP})_{\Sigma} \wedge J d(\mathrm{WP})_{\Sigma}
$$

where the measure for the open string is explicitly written as

$$
d(W P)_{\Sigma}=\frac{\operatorname{det}\left\langle S_{i} \mid \mu_{j}\right\rangle}{\left(\operatorname{det}\left\langle S_{i} \mid S_{j}\right\rangle\right)^{1 / 2}} \prod_{l=1}^{3 g-3} d m_{l}
$$

with $\mu_{i}$ the Beltrami differentials.

Finally we have to take care of the solitonic sum in (C.3). Since $I_{*}$ acts on the homology cycles of $\bar{\Sigma}$, the same will be true for the winding numbers of these cycles around the compact target dimension

$$
I_{*} N_{i}=\Gamma_{i j} N_{j}, \quad I_{*} M_{i}=-\Gamma_{j i} M_{j} .
$$

Because we are dividing by $I_{*}$, we are interested in retaining only those winding configurations that are symmetric under the involution. This implies that we have to truncate the solitonic sum down to those winding numbers satisfying $\Gamma \vec{N}=\vec{N}$ and $\Gamma^{\mathrm{t}} \vec{M}=-\vec{M}$ :

$$
\sum_{\text {solitons }} e^{-S_{c l}^{\text {open }}}=\sum_{\vec{N}, \vec{M}} \delta(\Gamma \vec{N}-\vec{N}) \delta\left(\Gamma^{\mathrm{t}} \vec{M}+\vec{M}\right) e^{-\frac{1}{2} S_{c \ell}^{\text {closed }}(\vec{N}, \vec{M})}
$$

The factor $\frac{1}{2}$ in front of the classical action arises because the area of the doubled surface is twice the area of the original bordered surface $\Sigma$ and therefore the classical action for open strings is half that for the auxiliary closed string theory.

By using the base in which $\Gamma$ is antidiagonal, it is straightforward to check that the winding numbers of the $A_{i}$ and $B_{i}$ cycles are respectively of the form

$$
\begin{aligned}
& \vec{N}=\left(n_{1}, \ldots, n_{h} ; q_{1}, \ldots, q_{p-1} ; n_{1} \ldots, n_{h}\right) \\
& \vec{M}=\left(m_{1}, \ldots, m_{h} ; 0, \ldots, 0 ;-m_{1}, \ldots,-m_{h}\right) .
\end{aligned}
$$

An overall change of sign in $\Gamma$ amounts to an interchange of $A$ with $B$ cycles (modular transformation) and therefore the structure of the winding numbers also gets interchanged. 
In the remainder of this Appendix we will use the techniques reviewed so far to compute the temperature-dependent divergences associated with the boundary of the moduli space of higher-order Riemann surfaces with boundaries. Let us assume that we have a genus- $h$ Riemann surface with $N$ boundaries attached to a $p$-dimensional D-brane 22 . Using what we have learned, we can write the formal contribution of such a diagram in terms of determinants evaluated on the doubled surface as

$$
\Lambda_{h, N}=\int_{\mathcal{M}(\Sigma)} d(\mathrm{WP})_{\Sigma} \sqrt{\left(\operatorname{det}\left(P^{+} P\right)_{\bar{\Sigma}}\right)^{1 / 2}\left[\frac{8 \pi^{2} \operatorname{det}^{\prime} \Delta_{\bar{\Sigma}}}{\int_{\bar{\Sigma}} d^{2} \xi \sqrt{g}}\right]^{-13}} R_{\bar{\Sigma}, I}^{\frac{p-12}{2}} \sum_{\text {solitons }} e^{-S_{c \ell}},
$$

where the square root is understood as a holomorphic square root. Divergences in $\Lambda_{h, N}$ will be associated with the boundary of the moduli space $\mathcal{M}(\Sigma)$; since we are interested in temperature-dependent singularities we can obviate any singular behaviour triggered by zero-momentum states. This means that we can focus our attention in two of the components of the boudary of $\mathcal{M}(\Sigma)$ : the shrinking of a boundary or the degeneration of a closed string handle.

Let us go first to the degeneration of a boundary. From (C.1) we see that this corresponds, for example, to the limit $\operatorname{Im} \tau_{h+1, h+1}=t_{11} \rightarrow \infty$. It can easily be checked that none of the entries of $(\operatorname{Im} \tau)^{-1}$ diverges in that limit, so from (C.5) we conclude that

$$
\sum_{\text {solitons }} e^{-S_{c \ell}} \sim 1+2 e^{-\frac{\beta^{2} t_{11}}{8 \pi \alpha^{\prime}}}+\ldots
$$

Having analysed the sum over classical vacua we turn to the path integral measure. Now we take advantage of having written the open string measure as, essentially, the square root of the closed string measure on the doubled surface and, using Belavin-Knizhnik theorem [45], we extract the limit

$$
\sqrt{\left(\operatorname{det}\left(P^{+} P\right)_{\bar{\Sigma}}\right)^{1 / 2}\left[\frac{8 \pi^{2} \operatorname{det}^{\prime} \Delta_{\bar{\Sigma}}}{\int_{\bar{\Sigma}} d^{2} \xi \sqrt{g}}\right]^{-13}} \sim\left|e^{2 \pi i \tau_{h+1, h+1}}\right|=e^{2 \pi t_{11}}
$$

This limit, together with the large $t_{11}$ expansion of the solitonic part, implies that the critical temperature is $\beta_{c}=4 \pi \sqrt{\alpha^{\prime}}$, in perfect accordance with the one-loop result.

22 In what follows we will consider that the compactified Euclidean time is always a Neumann direction and therefore $-1<p \leq 25$. Later on we will describe how to construct the D-instanton case $p=-1$ from our expressions. 
To complete the computation we have to study those divergences associated with the degeneration of a closed string handle. Now, however, because of the form of the period matrix (C.1), this limit corresponds to the degeneration of two handles in $\bar{\Sigma}$, those associated with the entries $\tau_{11}$ and $\tau_{h+N, h+N}$. Again it is very easy to find the asymptotic behaviour of the solitonic part in the $\operatorname{limit} \operatorname{Im} \tau_{11}=\operatorname{Im} \tau_{h+N, h+N} \equiv a \rightarrow \infty$ as

$$
\sum_{\text {solitons }} e^{-S_{c \ell}} \sim 1+2 e^{-\frac{\beta^{2} a}{4 \pi \alpha^{\prime}}}+\ldots
$$

Notice the additional factor of 2 in the exponent with respect to (C.6), due to the fact that we are degenerating two handles at a time. The Belavin-Knizhnik theorem comes again to the rescue in the computation of the limit of the string measure to find

$$
\sqrt{\left(\operatorname{det}\left(P^{+} P\right)_{\bar{\Sigma}}\right)^{1 / 2}\left[\frac{8 \pi^{2} \operatorname{det}^{\prime} \Delta_{\bar{\Sigma}}}{\int_{\bar{\Sigma}} d^{2} \xi \sqrt{g}}\right]^{-13}} \sim e^{4 \pi a},
$$

where again we have to keep in mind that we have two soft handles. Thus comparing the two exponential factors we come to the same value of the critical inverse temperature, $\beta_{c}=4 \pi \sqrt{\alpha^{\prime}}$.

We close our discussion with some remarks on the D-instanton case. As we already pointed out, in our framework the compact direction always has Neumann boundary conditions and this excludes the degenerate case $p=-1$. To recover the D-instanton we must begin with the D-particle $(p=0)$ and perform a Poisson resummation of the sum over classical vacua followed by a T-duality $\beta \rightarrow 4 \pi^{2} \alpha^{\prime} / \beta$ (for a discussion including higher-genus surfaces, see [46]). Following the same line of reasoning, we find the critical temperature $\beta_{c}=2 \pi \sqrt{\alpha^{\prime}}$ for all diagrams in perturbation theory.

We have concluded that higher-order diagrams do not modify the one-loop value of the critical length $\beta_{c}$ at which tachyon-triggered singularities appear; in this sense, the situation is pretty similar to that for closed strings [47], [48]. Whether or not this implies a non-renormalization of the Hagedorn temperature is much more difficult to decide, since non-trivial target-space effects may take place near the Hagedorn transition. In any case the situation is far from settled. 


\section{References}

[1] J. Polchinski, Rev. Mod. Phys. 68 (1996) 1245 (hep-th/9607050).

[2] J. Polchinski, Phys. Rev. Lett. 75 (1995) 4728 (hep-th/9510017).

[3] J. Dai, R.G. Leigh and J. Polchinski, Mod. Phys. Lett. A4 (1989) 2073.

[4] J. Polchinski, Phys. Rev. D50 (1994) 6041 (hep-th/9407031).

[5] S. Shenker, The strength of non-perturbative effects in String Theory, in: "Cargèse 1990, Proceedings, Random surfaces and quantum gravity".

[6] E.F. Corrigan and D.B. Fairlie, Nucl. Phys. B91 (1975) 527;

M.B. Green, Phys. Lett. B69 (1976) 89;

I.R. Klebanov and L. Thorlacius, Phys. Lett. B371 (1996) 51 (hep-th/9510200);

J.L.F. Barbón, Phys. Lett. B382 (1996) 60 (hep-th/9601098).

[7] A.M. Polyakov, Nucl. Phys. B120 (1977) 429.

[8] M.B. Green, Phys. Lett. B282 (1992) 380 (hep-th/9201054).

[9] E. Álvarez and M.A.R. Osorio, Physica A158 (1989) 449.

[10] M. Laucelli Meana, M.A.R. Osorio and J. Puente Peñalba, The string density of states from the convolution theorem, FFUOV-97/01 (hep-th/9701122).

[11] M.B. Green, Nucl. Phys. B381 (1992) 201.

[12] M.A. Vázquez-Mozo, Phys. Lett. B388 (1996) 494 (hep-th/9607052).

[13] G.W. Gibbons, M.B. Green and M.J. Perry, Phys. Lett. B370 (1996) 37 (hepth/9511080).

[14] G. 't Hooft, Phys. Rev. D14 (1976) 3432.

[15] M.B. Green, Phys. Lett. B354 (1995) 271 (hep-th/9504108).

[16] I. Affleck, Nucl. Phys. B191 (1981) 429.

[17] M. Shifman, Ed., Instantons in Gauge Theories (World Scientific, Singapore, 1994) p. 237-246.

[18] M.B. Green, Phys. Lett. B329 (1994) 435 (hep-th/9403040).

[19] J.M. Maldacena, Black holes in string theory, Princeton University Ph.D. thesis (hepth/9607235).

[20] J. Polchinski, TASI lectures on D-branes, NSF-ITP-96-145 (hep-th/9611050).

[21] J.L.F. Barbón, Nucl. Phys. B452 (1996) 313 (hep-th/9506137).

[22] J.L.F. Barbón, Remarks on the Classical Size of D-Branes, CERN-TH/97-52 (hepth/9703138). 
[23] T. Banks and L. Susskind, Brane-antibrane forces, RU-95-87 (hep-th/9511194).

[24] J.L.F. Barbón, Fermion exchange between D-instantons, CERN-TH/96-360 (hepth/9701075).

[25] E. Witten, Nucl. Phys. B460 (1996) 335 (hep-th/9510135).

[26] M.B. Green and M. Gutperle, Configurations of two D-instantons, DAMTP-96-110 (hep-th/9612127).

[27] M.B. Green and M. Gutperle, Effects of D-instantons, DAMTP-96-104 (hep-th/9701093).

[28] N. Seiberg and S. Shenker, Phys. Rev. D45 (1992) 4581 (hep-th/9201017).

[29] N. Cabibbo and G. Parisi, Phys. Lett. B59 (1975) 67.

[30] J. Atick and E. Witten, Nucl. Phys. B310 (1988) 291.

[31] S. Frautschi, Phys. Rev. D3 (1971) 2821.

[32] R.D. Carlitz, Phys. Rev. D5 (1972) 3231.

[33] E. Álvarez, Nucl. Phys. B269 (1986) 596.

[34] N. Deo, S. Jain and C.-I. Tan, Phys. Lett. B220 (1989) 125.

[35] J. Polchinski, Commun. Math. Phys. 104 (1986) 37.

[36] E. Álvarez and M.A.R. Osorio, Phys. Rev. D36 (1987) 1175.

[37] J. Minahan, Nucl. Phys. B333 (1990) 525.

[38] K. Hamada, Vertex operators for super-Yang-Mills and multi-D-branes in GreenSchwarz superstring, KEK-TH-504 (hep-th/9612234).

[39] V.A. Novikov, M.A. Shifman, A.I. Vainshtein, V.B. Voloshin and V.I. Zakharov, Nucl. Phys. B229 (1983) 394.

[40] M.A.R. Osorio and M.A. Vázquez-Mozo, Phys. Lett. B280 (1992) 21 (hepth/9201044); Phys. Rev. D47 (1993) 3411 (hep-th/9207002).

[41] C.P. Burgess and T.R. Morris, Nucl. Phys. B291 (1987) 256; Nucl. Phys. B291 (1987) 285;

A. Morozov and A. Rosly, Phys. Lett. B214 (1988) 522; Nucl. Phys. B326 (1989) 185; Nucl. Phys. B326 (1989) 205;

I.D. Vaisburg, Mod. Phys. Lett. A3 (1988) 511.

[42] S.K. Blau, M. Clements, S. Della Pietra, S. Carlip and V. Della Pietra, Nucl. Phys. B301 (1988) 285.

[43] M. Gutperle, Nucl. Phys. B444 (1995) 487 (hep-th/9502106).

[44] E. D'Hoker and D.H Phong, Rev. Mod. Phys. 60 (1988) 917. 
[45] A.A. Belavin and V.G. Knizhnik, Sov. Phys. JEPT 64 (1986) 214.

[46] E. Álvarez, J.L.F. Barbón and J. Borlaf, Nucl. Phys. B479 (1996) 218 (hepth/9603089).

[47] E. Álvarez and T. Ortín, Phys. Lett. B241 (1990) 215.

[48] E. Álvarez, T. Ortín and M.A.R. Osorio, Phys. Rev. D43 (1990) 3990. 